\title{
Damped response theory description of two-photon absorption
}

\author{
Kasper Kristensen, ${ }^{1, \text { a) }}$ Joanna Kauczor, ${ }^{1}$ Andreas J. Thorvaldsen, ${ }^{1, b}$ ) Poul Jørgensen, ${ }^{1}$ \\ Thomas Kjærgaard, ${ }^{2}$ and Antonio Rizzo ${ }^{3}$ \\ ${ }^{1}$ Lundbeck Foundation Center for Theoretical Chemistry, Department of Chemistry, Aarhus University, \\ Langelandsgade 140, DK-8000 Aarhus C, Denmark \\ ${ }^{2}$ Department of Chemistry, Oslo university, Postbox 1033, Blindern, 0315 Oslo, Norway \\ ${ }^{3}$ Istituto per i Processi Chimico-Fisici del Consiglio Nazionale delle Ricerche (IPCF-CNR), \\ Area della Ricerca, via G. Moruzzi 1, I-56124 Pisa, Italy
}

(Received 31 March 2011; accepted 9 May 2011; published online 1 June 2011)

\begin{abstract}
Damped response theory is applied to the calculation of two-photon absorption (TPA) spectra, which are determined directly, at each frequency, from a modified damped cubic response function. The TPA spectrum may therefore be evaluated for selected frequency ranges, making the damped TPA approach attractive for calculations on large molecules with a high density of states, where the calculation of TPA using standard theory is more problematic. Damped response theory can also be applied to the case of intermediate state resonances, where the standard TPA expression is divergent. Both exact damped response theory and its application within density functional theory are discussed. The latter is implemented using an atomic-orbital based density matrix formulation, which makes the approach especially suitable for studies on large systems. A test preliminary study is presented for the TPA spectrum of R-(+)-1,1'-bi(2-naphtol). () 2011 American Institute of Physics. [doi:10.1063/1.3595280]
\end{abstract}

\section{INTRODUCTION}

A vast range of molecular properties may be evaluated from standard response functions and their residues. ${ }^{1}$ In standard response theory the response functions diverge whenever one or more of the optical frequencies equal an excitation energy leading to a nonphysical behavior for molecular properties in the resonance region. However, by introducing empirical damping terms into the standard response function expressions, the singularities of the response functions are effectively removed by extending the domain of the response functions to the complex plane. ${ }^{2-5}$ The resulting damped response functions are therefore well-behaved in the entire frequency range.

Norman et al. ${ }^{6}$ formulated damped response theory by adding an empirical damping term to the standard Ehrenfest equation. ${ }^{7}$ In a recent paper $^{8}$ we described an alternative approach, where empirical excited states lifetimes are introduced in terms of complex excitation energies. The damped response theory formulation by Norman et al. ${ }^{6}$ and the one presented by Kristensen et al. ${ }^{8}$ are equivalent. Both approaches describe that effectively damped response theory corresponds to introducing complex frequencies into the standard response equations.

Damped response theory provides empirically broadened molecular absorption spectra in arbitrary frequency ranges, which may be a non-trivial task using standard response theory techniques, ${ }^{1}$ where each excited state in the frequency range of interest must be addressed individually to determine an absorption spectrum. In particular, this makes the calcula-

\footnotetext{
a)Electronic mail: kasperk@chem.au.dk.

b) Current address: CTCC, Department of Chemistry, University of Troms $\varnothing$, N-9037 Troms $\varnothing$, Norway
}

tion of absorption spectra for large molecules - which typically have high excited state densities - highly intractable. In contrast, damped response theory does not focus on the individual states but directly yields a complete absorption spectrum in the frequency range of interest, ${ }^{8}$ making damped response theory a very useful tool for investigating absorption spectra for large molecules.

During the last few years damped response theory has been applied to calculate a wide variety of molecular properties. Damped linear response properties that have been addressed using damped response theory include one-photon absorption (OPA) spectra and dispersion coefficients, ${ }^{8-16}$ optical rotation and electronic circular dichroism spectra, ${ }^{17-21}$ $\mathrm{x}$-ray absorption and natural circular dichroism spectra, ${ }^{22-27}$ the dynamic dipole magnetizability, ${ }^{28}$ and relativistic linear response functions. ${ }^{29,30}$ Damped non-linear molecular properties described by quadratic response theory have been addressed, including Raman scattering, ${ }^{31-41}$ the electro-optical Kerr effect and second-harmonic generation, ${ }^{6}$ and magnetic circular dichroism. ${ }^{42-44}$ Damped response theory has also been applied in the context of calculating vibrational spectra. ${ }^{45-47}$

In this paper we use the damped response theory formalism presented in Ref. 8 to calculate two-photon absorption (TPA) spectra. Introduced by Goeppert-Mayer in $1931,{ }^{48}$ TPA was first studied experimentally about 50 years ago. ${ }^{49,50}$ Since then TPA has become a powerful tool in numerous fields of science and technology, due to its applications, among others, in 3D fluorescence microscopy, ${ }^{51-53}$ optical limiting, $, 52,54$ optical data storage, ${ }^{55,56}$ and $3 \mathrm{D}$ microfabrication. ${ }^{51}$ Twophoton (and more generally multi-photon) spectroscopy exhibits greater 3D spatial selectivity, higher resolution and penetration than usual one-photon absorption spectroscopy, 
leading in turn to reduced scattering loss, photo-bleaching, and background fluorescence effects. The latest developments in the field of TPA materials, with emphasis on both the experimental and theoretical aspects, and on the strategies for the design and characterization of efficient chromophores, are discussed in detail in recent reviews. ${ }^{57-59}$

The first fully $a b$ initio calculation of two-photon transition matrix elements in molecules was performed about 25 years ago. ${ }^{60}$ Modern response function theory was then established, ${ }^{1}$ and it became straightforward to evaluate TPA for approximate electronic structure models and without recourse to truncated sum-over-state approaches. Highly accurate $a b$ initio wave function correlated models have been used to evaluate TPA spectra, however, the application range for these studies has been limited to molecules of relatively small size. The prediction of TPA cross sections needed for the modeling and design of strongly absorbing chromophores has therefore often been performed using semi-empirical methods often associated to effective few-state or exciton models, mainly aiming at a qualitative analysis. Today time-dependent density functional theory ${ }^{61-63}$ has become particularly useful being applicable to systems of ever increasing size and complexity.

In standard response theory the TPA amplitude may formally be determined from a residue analysis of the quadratic response function. ${ }^{1}$ However, the physical observable is proportional to the TPA strength (the amplitude squared). The latter equals a residue of the cubic response function, ${ }^{1}$ and it is therefore essential to consider damped cubic response theory to calculate damped TPA spectra. ${ }^{8}$

In this work we demonstrate an important difference between damped OPA and damped TPA. Whereas the damped OPA spectrum is identical to an absorption spectrum obtained by superimposing Lorentzian lineshape functions onto the standard absorption stick spectrum, ${ }^{8}$ the damped TPA spectrum generally differs somewhat from the standard TPA stick spectrum with superimposed lineshape functions. In the single-resonance case - where TPA may be envisioned as two consecutive one-photon transitions via a "virtual" state the standard and damped TPA spectra are virtually identical. However, if a double resonance is encountered - i.e., another excited state exists halfway between the ground state and the excited state under investigation - then the standard TPA expression diverges. In contrast, the damped TPA expression is well-defined and physically meaningful at all optical frequencies - regardless of the excited state spectrum.

The broadening of spectral lines has been discussed in the literature in great detail. The broadening arises from a large variety of physical phenomena such as ro-vibrational structure, Doppler broadening (thermal and inhomogeneous broadening), pressure (related to molecular collisions, depending on both the density and the temperature and often addressed as the major contribution to homogeneous broadening), and the fact that the excited state lifetimes are finite due to spontaneous emission. All these mechanisms are usually combined and supplemented by local and nonlocal mechanisms, related to the spatial extent of the experiment and to phenomena as self absorption, transit-time, saturation, and power broadening. ${ }^{64}$ With emphasis on nonlinear spectroscopies we refer to the classical textbooks of Mukamel, ${ }^{65}$ Shen, ${ }^{66}$ and Butcher and Cotter. ${ }^{67}$ The theory of collisional broadening in two-photon spectroscopy was laid down in Ref. 68, where the effects of inhomogeneous broadening, radiation damping, saturation, and intermediate resonant levels are also discussed. A discussion of how the TPA process is influenced by the ratio of the laser pulse duration compared to the excited state lifetime has also been presented. ${ }^{69}$ It is difficult - if at all possible to devise an accurate $a b$ initio model, which takes into account the very many different broadening effects and provides a $\gamma$ value tailored for each excited state. In any case such a task is beyond the scope of this paper, and we consider $\gamma$ as a single empirical parameter, effectively encompassing all the broadening phenomena listed above.

The outline for this paper is as follows. In Sec. II, we describe standard and damped TPA in exact response theory, and in Sec. III small test calculations of TPA spectra using KohnSham density functional theory (KS-DFT) are performed to compare standard and damped TPA spectra. Implementation details are given in Sec. IV. In Sec. V, we summarize the results of calculations on R-(+)-1,1'-bi(2-naphtol), or BINOL, a system which was recently the subject of a mixed experimental/theoretical study ${ }^{70-73}$ of its two-photon absorption and circular dichroism ${ }^{74,75}$ spectra. Section VI contains our conclusions and outlook.

\section{TWO-PHOTON ABSORPTION IN EXACT THEORY}

We consider the process where a molecular system in the ground state $|0\rangle$ undergoes a transition to the excited state $|n\rangle$ by absorbing two photons of frequencies $\omega_{\alpha}$ and $\omega_{\beta}$, i.e., $\omega_{\alpha}+\omega_{\beta}=\omega_{n}$, where $\hbar \omega_{n}$ is the $n$th excitation energy. We use atomic units throughout this paper (unless specified otherwise), i.e., $\hbar=e=m_{e}=a_{0}=1$. The $(a, b)$ th component of the two-photon transition amplitude tensor $T_{0 n}^{a b}\left(\omega_{\alpha}, \omega_{\beta}\right)$ may be written in the sum-over-states expression ${ }^{48,76}$

$$
T_{0 n}^{a b}\left(\omega_{\alpha}, \omega_{\beta}\right)=\sum_{p>0}\left(\frac{\mu_{0 p}^{a} \tilde{\mu}_{p n}^{b}}{\omega_{p}-\omega_{\alpha}}+\frac{\mu_{0 p}^{b} \tilde{\mu}_{p n}^{a}}{\omega_{p}-\omega_{\beta}}\right),
$$

where the sum is over all excited states (assumed to be real throughout the paper), $\mu^{a}$ and $\mu^{b}$ are components of the electric dipole operator, and where we have introduced the shorthand notations:

$$
\mu_{p q}^{a}=\left\langle p\left|\mu^{a}\right| q\right\rangle ; \quad \tilde{\mu}_{p q}^{a}=\mu_{p q}^{a}-\mu_{00}^{a} \delta_{p q} .
$$

For the remainder of this paper we assume that the two photons have the same energy, i.e., $\omega_{\alpha}=\omega_{\beta}=\omega$. In this case $T_{0 n}^{a b}(\omega, \omega)$ in Eq. (1) becomes,

$$
T_{0 n}^{a b}(\omega, \omega)=\sum_{p>0}\left(\frac{\mu_{0 p}^{a} \tilde{\mu}_{p n}^{b}}{\omega_{p}-\omega}+\frac{\mu_{0 p}^{b} \tilde{\mu}_{p n}^{a}}{\omega_{p}-\omega}\right),
$$

or, equivalently, since $2 \omega=\omega_{n}$,

$$
T_{0 n}^{a b}\left(\omega_{n} / 2, \omega_{n} / 2\right)=\sum_{p>0}\left(\frac{\mu_{0 p}^{a} \tilde{\mu}_{p n}^{b}}{\omega_{p}-\omega_{n} / 2}+\frac{\mu_{0 p}^{b} \tilde{\mu}_{p n}^{a}}{\omega_{p}-\omega_{n} / 2}\right) .
$$


The isotropically averaged expression for the TPA strength $\bar{\delta}_{0 n}$ in a sample of randomly tumbling molecules is given by ${ }^{76}$

$$
\begin{aligned}
\bar{\delta}_{0 n}= & \frac{1}{30} \sum_{a, b}\left\{F T_{0 n}^{a a}\left(\omega_{n} / 2, \omega_{n} / 2\right) T_{0 n}^{b b}\left(\omega_{n} / 2, \omega_{n} / 2\right)\right. \\
& \left.+(G+H) T_{0 n}^{a b}\left(\omega_{n} / 2, \omega_{n} / 2\right) T_{0 n}^{a b}\left(\omega_{n} / 2, \omega_{n} / 2\right)\right\},
\end{aligned}
$$

where the sums run independently over the $x, y$, and $z$ components of the molecular axes, and the numbers $F, G$, and $H$ depend on the polarization of the incident photons. In all results presented in this paper we consider the case, where both photons are linearly polarized with parallel propagation corresponding to $F=G=H=2{ }^{76}$

To evaluate Eq. (5), we need to determine a set of TPA strength tensor components $T_{0 n}^{a b}\left(\omega_{n} / 2, \omega_{n} / 2\right) T_{0 n}^{c d}\left(\omega_{n} / 2, \omega_{n} / 2\right)$, which may be obtained from a single residue of the cubic response function, ${ }^{1}$ as we discuss in Sec. II A below. If a lower lying excited state $|m\rangle$ happens to be exactly halfway between the ground state and $|n\rangle$ - i.e., $\omega_{m}=\omega_{n} / 2$ - then the standard expression for $T_{0 n}^{a b}\left(\omega_{n} / 2, \omega_{n} / 2\right)$ diverges, see Eq. (4). A phenomenological solution to this problem is to let the $m$ th excited state decay exponentially in time by introducing a finite excited state lifetime. ${ }^{2-5}$ Damped response theory provides a systematic way of doing this as discussed in Ref. 8. In Sec. II B, we describe the determination of TPA using damped response theory.

\section{A. Two-photon absorption in standard response theory}

We use the following index convention:

- abcdef denote combined frequency/operator-indices.

- kmn are positive indices labeling excitation energies and excited states.

- $p q r$ denote excitation energies and excited states and may be either positive or negative.

The TPA strength component $T_{0 n}^{a b}\left(\omega_{n} / 2, \omega_{n} / 2\right)$ $T_{0 n}^{c d}\left(\omega_{n} / 2, \omega_{n} / 2\right)$ may be determined from the residue of the cubic response function at $\omega_{c d}=\omega_{n}$ as shown, e.g., in Refs. 1 and 8. However, to simplify the following analysis we now carry out an alternative residue analysis of the cubic response function, which is more suitable for a generalization to damped response theory.

\section{Quasienergy formulation of response theory}

In the quasienergy formulation of response theory, the response functions are obtained as perturbation strength derivatives of the time-averaged quasienergy $\{Q\}_{T},{ }^{77,78}$

$$
\{Q\}_{T}=\int_{-T / 2}^{T / 2}\left\langle\tilde{0}\left|H-i \frac{\partial}{\partial t}\right| \tilde{0}\right\rangle d t,
$$

where $|\tilde{0}\rangle$ is the phase-isolated wave function for the perturbed molecular system and $H$ is the total Hamiltonian,

$$
H=H_{0}+V^{t},
$$

written as a sum of the Hamiltonian for the unperturbed system $H_{0}$ and a time-dependent harmonic perturbation $V^{t}$ oscillating with a period $T$,

$$
V^{t}=\sum_{b} e^{-i \omega_{b} t} \varepsilon_{b} \mu_{b}
$$

Above $\mu_{b}$ is a component of the electric operator and $\varepsilon_{b}$ is the corresponding electric field strength. The sum-index $b$ in Eq. (8) is a combined frequency/operator-index, ${ }^{8}$ which runs over both positive and negative frequencies and also over all operator components $x, y$, and $z$. The ensure the Hermicity of $V^{t}$, it is required that

$$
\varepsilon_{-b}=\varepsilon_{b}^{*} ; \quad \omega_{-b}=-\omega_{b} .
$$

We describe the perturbed wave function $|\tilde{0}\rangle$ in terms of a set of time-dependent response parameters and collect these in a vector $\mathbf{x}(t)$ which is expanded in orders of the perturbation,

$$
\mathbf{x}(t)=\mathbf{x}^{(1)}(t)+\mathbf{x}^{(2)}(t)+\ldots
$$

The individual elements in this order expansion may be expanded in terms of the different frequency and operator components present in $V^{t}$, e.g., the first- and second-order response vectors are written in the forms,

$$
\mathbf{x}^{(1)}(t)=\sum_{b} e^{-i \omega_{b} t} \varepsilon_{b} \mathbf{x}^{b},
$$

$$
\mathbf{x}^{(2)}(t)=\frac{1}{2 !} \sum_{b, c} e^{-i \omega_{b c} t} \varepsilon_{b} \varepsilon_{c} \mathbf{x}^{b c}
$$

We furthermore assume that the response parameters in the frequency domain are symmetric, e.g., $\mathbf{x}^{b c}=\mathbf{x}^{c b}$.

It is convenient to introduce the short-hand notations, ${ }^{79}$

$$
\begin{gathered}
Q^{a b \ldots f}=\left.\frac{d^{f}\{Q\}_{T}}{d \varepsilon_{a} d \varepsilon_{b} \cdots d \varepsilon_{f}}\right|_{\{\varepsilon\}=0}, \\
\mathbf{Q}^{N, 0}=\left.\frac{\partial^{N}\{Q\}_{T}}{\partial \mathbf{x}^{N}(t)}\right|_{\{\varepsilon\}=0}, \\
\mathbf{Q}^{N, a b \ldots f}=\left.\frac{\partial^{N+f}\{Q\}_{T}}{\partial \mathbf{x}^{N}(t) \partial \varepsilon_{a} \partial \varepsilon_{b} \cdots \partial \varepsilon_{f}}\right|_{\{\varepsilon\}=0},
\end{gathered}
$$

where $\{\varepsilon\}$ refers to the set of all perturbation strengths. We note that $Q^{a b \ldots f}$ represents the $f$ th order response function when the sum of frequencies is zero, ${ }^{77}$ i.e., $\omega_{a}+\omega_{b}+\ldots+$ $\omega_{f}=0$.

By differentiating the variational criteria for the timeaveraged quasienergy, ${ }^{77}$

$$
\frac{\partial\{Q\}_{T}}{\partial \mathbf{x}}=\mathbf{0},
$$

with respect to the perturbation strengths and evaluating at $\{\varepsilon\}=0$, we may determine a set of response equations for the response parameters, e.g., the first- and second-order equations for $\mathbf{x}^{b}$ and $\mathbf{x}^{c d}$ may be expressed in the following 
manner,

$$
\begin{gathered}
-\mathbf{Q}^{2,0} \mathbf{x}^{b}=\mathbf{Q}^{1, b}=\mathbf{g}^{b}, \\
-\mathbf{Q}^{2,0} \mathbf{x}^{c d}=\mathbf{Q}^{2, c} \mathbf{x}^{d}+\mathbf{Q}^{2, d} \mathbf{x}^{c}+\mathbf{Q}^{3,0} \mathbf{x}^{c} \mathbf{x}^{d}=\mathbf{g}^{c d},
\end{gathered}
$$

where we have used Eqs. (11) and (12) and defined the firstand second-order right-hand side vectors $\mathbf{g}^{b}$ and $\mathbf{g}^{c d}$.

In Ref. 8, the first- and second-order equations in Eq. (14) were expressed as

$$
\begin{gathered}
\left(\mathbf{E}^{[2]}-\omega_{b} \mathbf{S}^{[2]}\right) \mathbf{x}^{b}=\mathbf{g}^{b}, \\
\left(\mathbf{E}^{[2]}-\omega_{c d} \mathbf{S}^{[2]}\right) \mathbf{x}^{c d}=\mathbf{g}^{c d},
\end{gathered}
$$

or, equivalently, in the diagonal representations

$$
\begin{aligned}
& \left(\begin{array}{c}
{ }^{1} x_{k}^{b} \\
{ }^{2} x_{-k}^{b}
\end{array}\right)=\left(\begin{array}{cc}
\left(\omega_{k}-\omega_{b}\right)^{-1} & 0 \\
0 & \left(\omega_{k}+\omega_{b}\right)^{-1}
\end{array}\right)\left(\begin{array}{c}
{ }^{1} g_{-k}^{b} \\
2 g_{k}^{b}
\end{array}\right), \\
& \left(\begin{array}{c}
{ }^{1} x_{k}^{c d} \\
{ }^{2} x_{-k}^{c d}
\end{array}\right)=\left(\begin{array}{cc}
\left(\omega_{k}-\omega_{c d}\right)^{-1} & 0 \\
0 & \left(\omega_{k}+\omega_{c d}\right)^{-1}
\end{array}\right)\left(\begin{array}{c}
{ }^{1} g_{-k}^{c d} \\
{ }^{2} g_{k}^{c d}
\end{array}\right),
\end{aligned}
$$

and explicit expressions for the first- and second-order righthand side vectors were found to be

$$
g_{-k}^{b}=-\mu_{k 0}^{b} ; \quad g_{k}^{b}=\mu_{0 k}^{b},
$$

and

$$
\mathbf{g}^{c d}=P_{c d}\left(\boldsymbol{\mu}^{c[2]} \mathbf{x}^{d}\right)=\boldsymbol{\mu}^{c[2]} \mathbf{x}^{d}+\boldsymbol{\mu}^{d[2]} \mathbf{x}^{c},
$$

where we have implicitly defined the permutation operator $P_{c d}$. Using the structure of $\mu_{p q}^{c[2]}$ (Ref. 8)

$$
\mu_{p q}^{c[2]}= \begin{cases}-\tilde{\mu}_{-p q}^{c} & \text { for } p<0, q>0 \\ -\tilde{\mu}_{-q p}^{c} & \text { for } p>0, q<0 \\ 0 & \text { otherwise }\end{cases}
$$

we may write Eq. (18) in the form

$$
g_{-k}^{c d}=T_{0 k}^{c d}\left(\omega_{c}, \omega_{d}\right) ; \quad g_{k}^{c d}=-T_{0 k}^{c d}\left(-\omega_{c},-\omega_{d}\right),
$$

where we have used Eqs. (3) and (16a). We thus recognize that the second-order right-hand side vector corresponds to the TPA amplitude vector. This prove will be very useful for the following analysis.

\section{Two-photon absorption in cubic response theory}

The TPA strength for the $|0\rangle \rightarrow|n\rangle$ transition may be identified as the residue at $\omega_{c d}=\omega_{n}$ for the cubic response function $\left\langle\left\langle\mu^{a} ; \mu^{b}, \mu^{c}, \mu^{d}\right\rangle\right\rangle_{\omega_{b}, \omega_{c}, \omega_{d}}$ as demonstrated, e.g., in Ref. 1. We now provide an alternative residue analysis of the cubic response function to obtain equations that are more easily generalized to damped response theory.

The cubic response function equals the fourth-order derivative of the time-averaged quasienergy $Q^{a b c d}$ subject to the condition that $\omega_{a}+\omega_{b}+\omega_{c}+\omega_{d}=0 .{ }^{77}$ According to the $2 n+1$ rule (see, e.g., Ref. 79) $Q^{a b c d}$ can be expressed in terms of all first-order response parameters $\left(x^{a}, x^{b}, x^{c}, x^{d}\right)$ and the three second-order parameters not referencing perturbation $a\left(x^{b c}, x^{b d}, x^{c d}\right)$. All terms present in the total fourthorder derivative $Q^{a b c d}$ not containing any of these seven response parameters may simply be omitted because they multiply zero. This is a direct consequence of the variational condition for the time-averaged quasienergy in Eq. (13). Using this information we can express the total cubic response function in the following way:

$$
\begin{aligned}
&\left\langle\left\langle\mu^{a} ; \mu^{b}, \mu^{c}, \mu^{d}\right\rangle\right\rangle_{\omega_{b}, \omega_{c}, \omega_{d}} \\
&=Q^{a b c d}\left(\mathbf{x}^{a}, \mathbf{x}^{b}, \mathbf{x}^{c}, \mathbf{x}^{d}, \mathbf{x}^{b c}, \mathbf{x}^{b d}, \mathbf{x}^{c d}\right) \\
&=\left(\mathbf{Q}^{2, a} \mathbf{x}^{b}+\mathbf{Q}^{2, b} \mathbf{x}^{a}+\mathbf{Q}^{3,0} \mathbf{x}^{a} \mathbf{x}^{b}\right) \mathbf{x}^{c d} \\
&+\left(\mathbf{Q}^{2, a} \mathbf{x}^{c}+\mathbf{Q}^{2, c} \mathbf{x}^{a}+\mathbf{Q}^{3,0} \mathbf{x}^{a} \mathbf{x}^{c}\right) \mathbf{x}^{b d} \\
&+\left(\mathbf{Q}^{2, a} \mathbf{x}^{d}+\mathbf{Q}^{2, d} \mathbf{x}^{a}+\mathbf{Q}^{3,0} \mathbf{x}^{a} \mathbf{x}^{d}\right) \mathbf{x}^{b c} \\
&+\mathbf{Q}^{3, a} \mathbf{x}^{b} \mathbf{x}^{c} \mathbf{x}^{d}+\mathbf{Q}^{3, b} \mathbf{x}^{a} \mathbf{x}^{c} \mathbf{x}^{d}+\mathbf{Q}^{3, c} \mathbf{x}^{a} \mathbf{x}^{b} \mathbf{x}^{d} \\
&+\mathbf{Q}^{3, d} \mathbf{x}^{a} \mathbf{x}^{b} \mathbf{x}^{c}+\mathbf{Q}^{4,0} \mathbf{x}^{a} \mathbf{x}^{b} \mathbf{x}^{c} \mathbf{x}^{d} \\
&= \mathbf{g}^{a b} \mathbf{x}^{c d}+\mathbf{g}^{a c} \mathbf{x}^{b d}+\mathbf{g}^{a d} \mathbf{x}^{b c} \\
&+\mathbf{Q}^{3, a} \mathbf{x}^{b} \mathbf{x}^{c} \mathbf{x}^{d}+\mathbf{Q}^{3, b} \mathbf{x}^{a} \mathbf{x}^{c} \mathbf{x}^{d}+\mathbf{Q}^{3, c} \mathbf{x}^{a} \mathbf{x}^{b} \mathbf{x}^{d} \\
&+\mathbf{Q}^{3, d} \mathbf{x}^{a} \mathbf{x}^{b} \mathbf{x}^{c}+\mathbf{Q}^{4,0} \mathbf{x}^{a} \mathbf{x}^{b} \mathbf{x}^{c} \mathbf{x}^{d},
\end{aligned}
$$

where we have used Eq. (14b) to obtain the final equality.

When identifying the residue of the cubic response function at $\omega_{c d}=\omega_{n}$ only terms in Eq. (21) containing $x_{n}^{c d}$ may contribute, see Eq. (16b). To evaluate the residue at $\omega_{c d}$ $=\omega_{n}$, it is therefore sufficient to consider a modified standard TPA response function $\left\langle\left\langle\mu^{a} ; \mu^{b}, \mu^{c}, \mu^{d}\right\rangle\right\rangle_{\omega_{b}, \omega_{c}, \omega_{d}}\left(x^{c d}\right)$, where only the terms in total cubic response function $\left\langle\left\langle\mu^{a} ; \mu^{b}, \mu^{c}, \mu^{d}\right\rangle\right\rangle_{\omega_{b}, \omega_{c}, \omega_{d}}$ containing $x^{c d}$ are kept since

$$
\begin{aligned}
& \lim _{\omega_{c d} \rightarrow \omega_{n}}\left(\omega_{c d}-\omega_{n}\right)\left\langle\left\langle\mu^{a} ; \mu^{b}, \mu^{c}, \mu^{d}\right\rangle\right\rangle_{\omega_{b}, \omega_{c}, \omega_{d}} \\
& =\lim _{\omega_{c d} \rightarrow \omega_{n}}\left(\omega_{c d}-\omega_{n}\right)\left\langle\left\langle\mu^{a} ; \mu^{b}, \mu^{c}, \mu^{d}\right\rangle\right\rangle_{\omega_{b}, \omega_{c}, \omega_{d}}\left(x^{c d}\right) .
\end{aligned}
$$

From Eq. (21), we see that only one term contains $x^{c d}$, and the standard TPA response function thus becomes

$$
\left\langle\left\langle\mu^{a} ; \mu^{b}, \mu^{c}, \mu^{d}\right\rangle\right\rangle_{\omega_{b}, \omega_{c}, \omega_{d}}\left(x^{c d}\right)=\mathbf{g}^{a b} \mathbf{x}^{c d}=\sum_{q} g_{q}^{a b} x_{q}^{c d},
$$

where the summation is over both positive and negative $q$ indices.

Alternatively we may use Eqs. (16b) and (20) to express Eq. (23) in the form,

$$
\begin{aligned}
& \left\langle\left\langle\mu^{a} ; \mu^{b}, \mu^{c}, \mu^{d}\right\rangle\right\rangle_{\omega_{b}, \omega_{c}, \omega_{d}}\left(x^{c d}\right) \\
& =-\sum_{q>0}\left\{\frac{T_{0 q}^{a b}\left(-\omega_{a},-\omega_{b}\right) T_{0 q}^{c d}\left(\omega_{c}, \omega_{d}\right)}{\omega_{q}-\omega_{c d}}\right. \\
& \left.\quad+\frac{T_{0 q}^{a b}\left(\omega_{a}, \omega_{b}\right) T_{0 q}^{c d}\left(-\omega_{c},-\omega_{d}\right)}{\omega_{q}+\omega_{c d}}\right\} .
\end{aligned}
$$


The form of $\left\langle\left\langle\mu^{a} ; \mu^{b}, \mu^{c}, \mu^{d}\right\rangle\right\rangle_{\omega_{b}, \omega_{c}, \omega_{d}}\left(x^{c d}\right)$ in Eq. (23) is useful from a computational point of view as will be discussed in detail in Sec. IV A, where we consider the implementation of TPA for density functional theory (DFT) response theory. We emphasize that to arrive at Eq. (23) we have only used the fact that the time-averaged quasienergy is variational. Equation (23) is therefore also valid in variational approximate electron structure theories and not only in exact theory. The alternative expression in Eq. (24) is useful for analysis purposes, which is our focus now. In particular, the residue of the cubic response function at $\omega_{c d}=\omega_{n}$ may now easily be identified,

$$
\begin{aligned}
& \lim _{\omega_{c d} \rightarrow \omega_{n}}\left(\omega_{c d}-\omega_{n}\right)\left\langle\left\langle\mu^{a} ; \mu^{b}, \mu^{c}, \mu^{d}\right\rangle\right\rangle_{\omega_{b}, \omega_{c}, \omega_{d}} \\
& \quad=\lim _{\omega_{c d} \rightarrow \omega_{n}}\left(\omega_{c d}-\omega_{n}\right)\left\langle\left\langle\mu^{a} ; \mu^{b}, \mu^{c}, \mu^{d}\right\rangle\right\rangle_{\omega_{b}, \omega_{c}, \omega_{d}}\left(x^{c d}\right) \\
& =T_{0 n}^{a b}\left(-\omega_{a},-\omega_{b}\right) T_{0 n}^{c d}\left(\omega_{c}, \omega_{d}\right)
\end{aligned}
$$

where $\omega_{c}+\omega_{d}=\omega_{n}$ and consequently $\omega_{a}+\omega_{b}=-\omega_{n}$. We may still consider $\omega_{a}$ (or $\omega_{b}$ ) and $\omega_{c}$ (or $\omega_{d}$ ) as independent parameters. By choosing $\omega_{c}=\omega$ and $\omega_{a}=-\omega$ (where $\omega$ is a (positive) optical laser frequency) we obtain the following frequency relations:

$\omega_{a}=\omega_{b}=-\omega ; \quad \omega_{c}=\omega_{d}=\omega ; \quad \omega_{c d}=\omega_{c}+\omega_{d}=2 \omega$,

and Eq. (25) becomes,

$$
\begin{gathered}
\lim _{2 \omega \rightarrow \omega_{n}}\left(2 \omega-\omega_{n}\right)\left\langle\left\langle\mu^{a} ; \mu^{b}, \mu^{c}, \mu^{d}\right\rangle\right\rangle_{-\omega, \omega, \omega} \\
\quad=T_{0 n}^{a b}\left(\omega_{n} / 2, \omega_{n} / 2\right) T_{0 n}^{c d}\left(\omega_{n} / 2, \omega_{n} / 2\right) .
\end{gathered}
$$

Thus, the residue of the cubic response function at $\omega_{c d}=\omega_{n}$ yields the $(a, b, c, d)$ th component of the TPA strength tensor $T_{0 n}^{a b}\left(\omega_{n} / 2, \omega_{n} / 2\right) T_{0 n}^{c d}\left(\omega_{n} / 2, \omega_{n} / 2\right)$ for the $|0\rangle \rightarrow|n\rangle$ transition.

We mention that the TPA amplitude component $T_{0 n}^{a b}\left(\omega_{n} / 2, \omega_{n} / 2\right)$ may also be determined from a residue of the quadratic response function. Specifically, the residue of the quadratic response function equals the product of a TPA amplitude and an OPA amplitude (a dipole matrix element): ${ }^{1}$

$$
\begin{aligned}
& \left.\left(\lim _{\omega_{c} \rightarrow \omega_{n}}\left(\omega_{c}-\omega_{n}\right)\left\langle\left\langle\mu^{a} ; \mu^{b}, \mu^{c}\right\rangle\right\rangle_{-\omega_{b}, \omega_{c}}\right)\right|_{\omega_{b}=\omega_{n} / 2} \\
& \quad=-T_{0 n}^{a b}\left(\omega_{n} / 2, \omega_{n} / 2\right) \mu_{n 0}^{c} .
\end{aligned}
$$

In standard response theory it is straightforward to separate the $T_{0 n}^{a b}\left(\omega_{n} / 2, \omega_{n} / 2\right)$ and $\mu_{n 0}^{c}$ contributions and subsequently evaluate the TPA strengths simply by squaring the TPA amplitudes, see Eq. (5). In damped quadratic response theory a spectrum of the residues in Eq. (28) with superimposed lineshape functions is obtained, ${ }^{8}$ and it is not possible to separate out the individual TPA and OPA amplitude components from this spectrum. However, the cubic residue in Eq. (25) directly equals the TPA strength, and the corresponding damped cubic response function therefore directly yields a spectrum of TPA strengths. We elaborate on this matter in Sec. II B below.
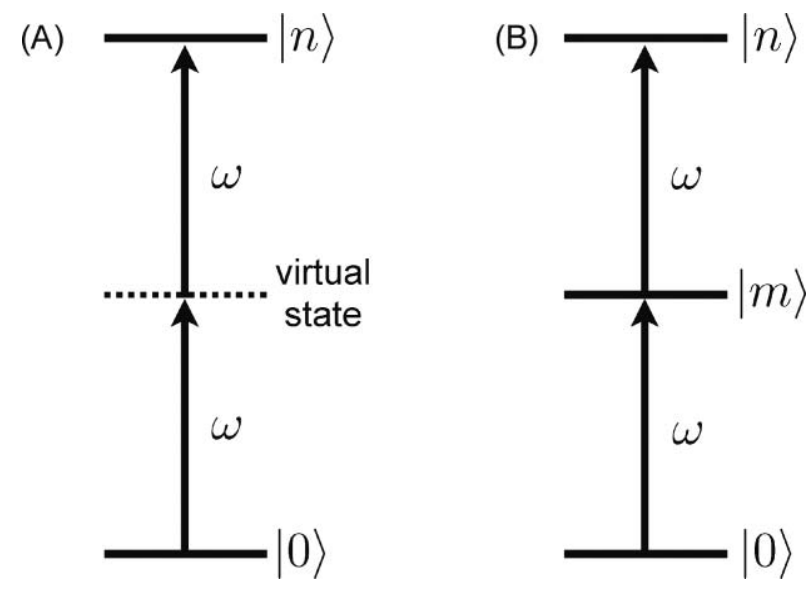

FIG. 1. Illustration of the TPA $|0\rangle \rightarrow|n\rangle$ transition via (a) a "virtual" state (single-resonance case), and (b) via an actual intermediate state $|m\rangle$ (doubleresonance case).

\section{Double-resonances in standard TPA theory}

The TPA transition $|0\rangle \rightarrow|n\rangle$ is usually interpreted as the absorption of one photon to a so-called "virtual" state, followed by a second one-photon transition from the virtual state to the final state $|n\rangle$ as depicted in Fig. 1(a). This interpretation is useful if $|n\rangle$ is one of the lower lying excited states.

If $|n\rangle$ is a higher lying excited state there might exist an intermediate state $|m\rangle$ halfway between the ground state $|0\rangle$ and $|n\rangle$ as depicted in Fig. 1(b), i.e., $\omega_{m}=\omega_{n} / 2$. If the laser frequency $\omega$ is chosen such that $\omega=\omega_{m}=\omega_{n} / 2$, the one-photon transitions $|0\rangle \rightarrow|m\rangle$ and $|m\rangle \rightarrow|n\rangle$ will be simultaneously resonant. We refer to this situation as a doubleresonance.

When $\omega=\omega_{m}=\omega_{n} / 2$, the $m$ th component in the expression for the TPA amplitude in Eq. (4) diverges. This divergence is caused by the fact that the excited state lifetimes in standard response theory are infinite. A standard way of treating this problem is to introduce a phenomenological lifetime for the excited states, which are close to resonance, see, e.g., Refs. 2-5. The introduction of a finite lifetime for the state $|m\rangle$ is equivalent to introducing a complex excitation energy. We refer to response theory where phenomenological lifetimes have been introduced as damped response theory. We now describe how damped response theory can be used to determine TPA spectra where the double-resonance case can also be treated.

\section{B. Two-photon absorption in damped response theory}

In the damped response theory formulation in Ref. 8 it was demonstrated that the introduction of effective excited state lifetimes in terms of complex excitation energies effectively corresponds to carrying out the replacements,

$$
\frac{1}{\omega_{m}-\omega_{\alpha}} \rightarrow \frac{1}{\omega_{m}-\left(\omega_{\alpha}+i \gamma\right)}=\mathcal{D}_{m}\left(\omega_{\alpha}\right)+i \mathcal{A}_{m}\left(\omega_{\alpha}\right),
$$




$$
\frac{1}{\omega_{m}+\omega_{\alpha}} \rightarrow \frac{1}{\omega_{m}+\left(\omega_{\alpha}+i \gamma\right)}=\mathcal{D}_{m}\left(-\omega_{\alpha}\right)-i \mathcal{A}_{m}\left(-\omega_{\alpha}\right),
$$

whenever terms of the form $\left(\omega_{m}-\omega_{\alpha}\right)^{-1}$ or $\left(\omega_{m}+\omega_{\alpha}\right)^{-1}$ occur in the standard expressions for response functions. In Eq. (29) $\omega_{m}$ is an excitation energy, $\omega_{\alpha}(\alpha=a, b, c, d$, or $c d$ in this work) is an optical frequency, and $\gamma=\frac{1}{2} \tau^{-1}$ is the empirical broadening parameter, where $\tau$ is the effective lifetime common to all excited states. The dispersion $\mathcal{D}$ and absorption $\mathcal{A}$ lineshape functions are given by

$$
\begin{aligned}
& \mathcal{D}_{m}(\omega)=\frac{\omega_{m}-\omega}{\left(\omega_{m}-\omega\right)^{2}+\gamma^{2}}, \\
& \mathcal{A}_{m}(\omega)=\frac{\gamma}{\left(\omega_{m}-\omega\right)^{2}+\gamma^{2}} .
\end{aligned}
$$

Equation (29) effectively corresponds to adding the imaginary factor $i \gamma$ to the (real) optical frequency $\omega_{\alpha}$. We note that $\gamma$ describes the half width at half maximum (HWHM) for the (non-normalized) Lorentzian $\mathcal{A}(\omega)$, and it is therefore a measure of the width of absorption peaks in damped absorption spectra. As discussed in the Introduction we consider $\gamma$ as an empirical parameter effectively encompassing a wide variety of broadening effects.

The first- and second-order damped response equations may now be obtained by carrying out the replacements in Eq. (29) into Eqs. (15) and (16) to obtain

$$
\left(\mathbf{E}^{[2]}-\left(\omega_{b}+i \gamma\right) \mathbf{S}^{[2]}\right) \overline{\mathbf{x}}^{b}=\mathbf{g}^{b},
$$

$$
\left(\mathbf{E}^{[2]}-\left(\omega_{c d}+i \gamma\right) \mathbf{S}^{[2]}\right) \overline{\mathbf{x}}^{c d}=\overline{\mathbf{g}}^{c d},
$$

and the diagonal representation

$$
\begin{aligned}
\left(\begin{array}{c}
{ }^{1} \bar{x}_{k}^{b} \\
{ }^{2} \bar{x}_{-k}^{b}
\end{array}\right)= & \left(\begin{array}{cc}
\left(\omega_{k}-\omega_{b}-i \gamma\right)^{-1} & 0 \\
0 & \left(\omega_{k}+\omega_{b}+i \gamma\right)^{-1}
\end{array}\right) \\
& \times\left(\begin{array}{c}
{ }^{1} g_{-k}^{b} \\
{ }^{2} g_{k}^{b}
\end{array}\right), \\
\left(\begin{array}{c}
{ }^{1} \bar{x}_{k}^{c d} \\
{ }^{2} \bar{x}_{-k}^{c d}
\end{array}\right)= & \left(\begin{array}{cc}
\left(\omega_{k}-\omega_{c d}-i \gamma\right)^{-1} & 0 \\
0 & \left(\omega_{k}+\omega_{c d}+i \gamma\right)^{-1}
\end{array}\right) \\
& \times\left(\begin{array}{c}
{ }^{1} \bar{g}_{-k}^{c d} \\
{ }^{2} \bar{g}_{k}^{c d}
\end{array}\right),
\end{aligned}
$$

where the (complex) damped quantities are denoted by bars. Note that the first-order right-hand side vector $g^{b}$ contains no excitation energies, see Eq. (17), and consequently $g^{b}$ is real (and, therefore, not denoted by a bar). In contrast, the complex damped second-order right-hand side vector $\bar{g}^{c d}$ is obtained by carrying out the replacement in Eq. (29) into Eq. (20):

$$
\begin{aligned}
\bar{g}_{-k}^{c d} & =\sum_{p>0}\left(\frac{\mu_{0 p}^{c} \tilde{\mu}_{p k}^{d}}{\omega_{p}-\omega_{c}-i \gamma}+\frac{\mu_{0 p}^{d} \tilde{\mu}_{p k}^{c}}{\omega_{p}-\omega_{d}-i \gamma}\right) \\
= & T_{0 k}^{c d}\left(\omega_{c}+i \gamma, \omega_{d}+i \gamma\right)=T_{0 k}^{c d}(\omega+i \gamma, \omega+i \gamma) \\
\bar{g}_{k}^{c d} & =-\sum_{p>0}\left(\frac{\mu_{0 p}^{c} \tilde{\mu}_{p k}^{d}}{\omega_{p}+\omega_{c}+i \gamma}+\frac{\mu_{0 p}^{d} \tilde{\mu}_{p k}^{c}}{\omega_{p}+\omega_{d}+i \gamma}\right) \\
& =-T_{0 k}^{c d}\left(-\omega_{c}-i \gamma,-\omega_{d}-i \gamma\right) \\
& =-T_{0 k}^{c d}(-\omega-i \gamma,-\omega-i \gamma)
\end{aligned}
$$

where we have used the frequency relations in Eq. (26) and allowed for complex frequency arguments in the TPA amplitude in Eq. (1), i.e.,

$$
\begin{aligned}
T_{0 n}^{c d}(\omega+i \gamma, \omega+i \gamma)= & \sum_{p>0}\left(\frac{\mu_{0 p}^{c} \tilde{\mu}_{p n}^{d}}{\omega_{p}-(\omega+i \gamma)}\right. \\
& \left.+\frac{\mu_{0 p}^{d} \tilde{\mu}_{p n}^{c}}{\omega_{p}-(\omega+i \gamma)}\right), \\
T_{0 n}^{c d}(-\omega-i \gamma,-\omega-i \gamma)= & \sum_{p>0}\left(\frac{\mu_{0 p}^{c} \tilde{\mu}_{p n}^{d}}{\omega_{p}-(-\omega-i \gamma)}\right. \\
& \left.+\frac{\mu_{0 p}^{d} \tilde{\mu}_{p n}^{c}}{\omega_{p}-(-\omega-i \gamma)}\right) .
\end{aligned}
$$

Similarly, the $\bar{g}^{a b}$ vector becomes,

$$
\begin{aligned}
\bar{g}_{-k}^{a b} & =T_{0 k}^{a b}\left(\omega_{a}+i \gamma, \omega_{b}+i \gamma\right) \\
& =T_{0 k}^{a b}(-\omega+i \gamma,-\omega+i \gamma),
\end{aligned}
$$

$$
\begin{aligned}
\bar{g}_{k}^{a b} & =-T_{0 k}^{a b}\left(-\omega_{a}-i \gamma,-\omega_{b}-i \gamma\right) \\
& =-T_{0 k}^{a b}(\omega-i \gamma, \omega-i \gamma)
\end{aligned}
$$

where

$$
\begin{aligned}
& T_{0 n}^{a b}(-\omega+i \gamma,-\omega+i \gamma) \\
&=\sum_{p>0}\left(\frac{\mu_{0 p}^{a} \tilde{\mu}_{p n}^{b}}{\omega_{p}-(-\omega+i \gamma)}+\frac{\mu_{0 p}^{b} \tilde{\mu}_{p n}^{a}}{\omega_{p}-(-\omega+i \gamma)}\right), \\
& T_{0 n}^{a b}(\omega-i \gamma, \omega-i \gamma)= \sum_{p>0}\left(\frac{\mu_{0 p}^{a} \tilde{\mu}_{p n}^{b}}{\omega_{p}-(\omega-i \gamma)}\right. \\
&\left.+\frac{\mu_{0 p}^{b} \tilde{\mu}_{p n}^{a}}{\omega_{p}-(\omega-i \gamma)}\right) .
\end{aligned}
$$


Thus, if $a=c$ and $b=d$, the $\bar{g}^{a b}$ and $\bar{g}^{c d}$ vectors are related in the following simple manner

$$
\bar{g}_{-q}^{a b}=-\left(\bar{g}_{q}^{c d}\right)^{*} \quad(a=c, b=d),
$$

which is valid for both positive and negative $q$ indices.

Expressions for the standard TPA response function were given in Eqs. (23) and (24). Corresponding expressions for the damped TPA response function $\left\langle\left\langle\overline{\mu^{a} ; \mu^{b}, \mu^{c}, \mu^{d}}\right\rangle\right\rangle_{\omega_{b}, \omega_{c}, \omega_{d}}\left(\bar{x}^{c d}\right)$ can be identified by replacing all standard quantities by their damped counterparts (e.g., replacing $g^{c d}$ by $\bar{g}^{c d}$ ) to obtain the damped counterparts of Eqs. (23) and (24):

$$
\left\langle\left\langle\overline{\mu^{a} ; \mu^{b}, \mu^{c}, \mu^{d}}\right\rangle\right\rangle_{\omega_{b}, \omega_{c}, \omega_{d}}\left(\bar{x}^{c d}\right)=\sum_{q} \bar{g}_{q}^{a b} \bar{x}_{q}^{c d},
$$

$$
\begin{array}{r}
\left\langle\left\langle\overline{\mu^{a} ; \mu^{b}, \mu^{c}, \mu^{d}}\right\rangle\right\rangle_{\omega_{b}, \omega_{c}, \omega_{d}}\left(\bar{x}^{c d}\right)=-\sum_{q>0} \\
\quad\left\{\frac{T_{0 q}^{a b}\left(-\omega_{a}-i \gamma,-\omega_{b}-i \gamma\right) T_{0 q}^{c d}\left(\omega_{c}+i \gamma, \omega_{d}+i \gamma\right)}{\omega_{q}-\omega_{c d}-i \gamma}\right. \\
\left.+\frac{T_{0 q}^{a b}\left(\omega_{a}+i \gamma, \omega_{b}+i \gamma\right) T_{0 q}^{c d}\left(-\omega_{c}-i \gamma,-\omega_{d}-i \gamma\right)}{\omega_{q}+\omega_{c d}+i \gamma}\right\} \\
=-\sum_{q>0}\left\{\frac{T_{0 q}^{a b}(\omega-i \gamma, \omega-i \gamma) T_{0 q}^{c d}(\omega+i \gamma, \omega+i \gamma)}{\omega_{q}-2 \omega-i \gamma}\right. \\
\left.+\frac{T_{0 q}^{a b}(-\omega+i \gamma,-\omega+i \gamma) T_{0 q}^{c d}(-\omega-i \gamma,-\omega-i \gamma)}{\omega_{q}+2 \omega+i \gamma}\right\},
\end{array}
$$

where we have inserted the frequency relations in Eq. (26) to obtain the last equality in Eq. (39). Equation (38) is useful from a computational point of view and will be discussed in Sec. IV B, whereas Eq. (39) is useful for analysis purposes which is our focus now.

We note that the norm of the denominator in the first term of Eq. (39) is much smaller than the norm of the denominator in the second term since $\omega>0$. Similarly, from Eqs. (34) and (36) it follows that the norm of the damped TPA amplitudes in the first term is much larger than those in the second term,

$$
\begin{aligned}
& \left|T_{0 q}^{a b}(\omega-i \gamma, \omega-i \gamma)\right| \gg\left|T_{0 q}^{a b}(-\omega+i \gamma,-\omega+i \gamma)\right|, \\
& \left|T_{0 q}^{c d}(\omega+i \gamma, \omega+i \gamma)\right| \gg\left|T_{0 q}^{c d}(-\omega-i \gamma,-\omega-i \gamma)\right| .
\end{aligned}
$$

Thus, the first term in Eq. (39) completely dominates the expression, and for analysis purposes we may omit the second term,

$$
\begin{aligned}
& \left\langle\left\langle\overline{\mu^{a} ; \mu^{b}, \mu^{c}, \mu^{d}}\right\rangle\right\rangle_{\omega_{b}, \omega_{c}, \omega_{d}}\left(\bar{x}^{c d}\right) \\
& \approx-\sum_{q>0} \frac{T_{0 q}^{a b}(\omega-i \gamma, \omega-i \gamma) T_{0 q}^{c d}(\omega+i \gamma, \omega+i \gamma)}{\omega_{q}-2 \omega-i \gamma} .
\end{aligned}
$$

We now demonstrate that the isotropic average of the imaginary components of the numerator in Eq. (41) vanishes, which will simplify the following analysis. The isotropic average in Eq. (5) contains two types of components: (i) $a b a b$ and (ii) $a a b b$. For the $a b a b$-components we obtain, using Eqs. (34a) and (36b),

$$
\begin{aligned}
& T_{0 q}^{a b}(\omega-i \gamma, \omega-i \gamma) T_{0 q}^{a b}(\omega+i \gamma, \omega+i \gamma) \\
& \quad=T_{0 q}^{a b}(\omega+i \gamma, \omega+i \gamma)^{*} T_{0 q}^{a b}(\omega+i \gamma, \omega+i \gamma) \\
& \quad=\left|T_{0 q}^{a b}(\omega+i \gamma, \omega+i \gamma)\right|^{2} .
\end{aligned}
$$

Equation (42) states that the $a b a b$-components of the damped TPA strengths are purely real. For the $a a b b$-components we need to consider the imaginary part of the full sum in Eq. (5),

$$
\begin{aligned}
& \operatorname{Im} \sum_{a, b}\left\{T_{0 q}^{a a}(\omega-i \gamma, \omega-i \gamma) T_{0 q}^{b b}(\omega+i \gamma, \omega+i \gamma)\right\} \\
& =\operatorname{Im} \sum_{a>b}\left\{T_{0 q}^{a a}(\omega-i \gamma, \omega-i \gamma) T_{0 q}^{b b}(\omega+i \gamma, \omega+i \gamma)\right. \\
& \left.\quad+T_{0 q}^{b b}(\omega-i \gamma, \omega-i \gamma) T_{0 q}^{a a}(\omega+i \gamma, \omega+i \gamma)\right\} \\
& =\operatorname{Im} \sum_{a>b}\left\{T_{0 q}^{a a}(\omega-i \gamma, \omega-i \gamma) T_{0 q}^{b b}(\omega+i \gamma, \omega+i \gamma)\right. \\
& \left.\quad+\left[T_{0 q}^{a a}(\omega-i \gamma, \omega-i \gamma) T_{0 q}^{b b}(\omega+i \gamma, \omega+i \gamma)\right]^{*}\right\}=0,
\end{aligned}
$$

where we have used Eq. (42) for $a=b$ to remove the diagonal terms, and also the fact that the sum of a complex number and its complex conjugate is purely real.

In conclusion, when calculating the isotropic average in Eq. (5) for the damped TPA strengths the imaginary components are either zero term by term ( $a b a b$-components), or 
cancel each other when the summation is carried out ( $a a b b$ components). Thus, only the real parts of the damped TPA strength components contribute to the physical observable TPA and we, therefore, disregard the imaginary part in the following analysis.

Let us write out the $(a, b, c, d)$ th component of the damped TPA strength tensor in Eq. (41) and leave out the redundant imaginary part,

$$
\begin{aligned}
& T_{0 q}^{a b}(\omega-i \gamma, \omega-i \gamma) T_{0 q}^{c d}(\omega+i \gamma, \omega+i \gamma) \\
& =P_{a b}\left[\sum_{p>0} \frac{\mu_{0 p}^{a} \tilde{\mu}_{p q}^{b}}{\omega_{p}-\omega+i \gamma}\right] P_{c d}\left[\sum_{r>0} \frac{\mu_{0 r}^{c} \tilde{\mu}_{r q}^{d}}{\omega_{r}-\omega-i \gamma}\right] \\
& =P_{a b} P_{c d} \sum_{p>0, r>0}\left\{\mu _ { 0 p } ^ { a } \tilde { \mu } _ { p q } ^ { b } \mu _ { 0 r } ^ { c } \tilde { \mu } _ { r q } ^ { d } \left[\mathcal{D}_{p}(\omega) \mathcal{D}_{r}(\omega)\right.\right. \\
& \left.\left.\quad+\mathcal{A}_{p}(\omega) \mathcal{A}_{r}(\omega)\right]\right\} \\
& =\Phi_{q}^{a b c d}(\omega)+\Lambda_{q}^{a b c d}(\omega),
\end{aligned}
$$

where we have used Eq. (30) and introduced the damped TPA strength functions $\Phi_{q}^{a b c d}(\omega)$ and $\Lambda_{q}^{a b c d}(\omega)$, which are both purely real,

$$
\Phi_{q}^{a b c d}(\omega)=P_{a b} P_{c d} \sum_{p>0, r>0} \mu_{0 p}^{a} \tilde{\mu}_{p q}^{b} \mu_{0 r}^{c} \tilde{\mu}_{r q}^{d} \mathcal{D}_{p}(\omega) \mathcal{D}_{r}(\omega),
$$

$$
\Lambda_{q}^{a b c d}(\omega)=P_{a b} P_{c d} \sum_{p>0, r>0} \mu_{0 p}^{a} \tilde{\mu}_{p q}^{b} \mu_{0 r}^{c} \tilde{\mu}_{r q}^{d} \mathcal{A}_{p}(\omega) \mathcal{A}_{r}(\omega)
$$

Inserting Eq. (44) into Eq. (41) we obtain

$$
\begin{aligned}
& \left\langle\left\langle\overline{\left.\mu^{a} ; \mu^{b}, \mu^{c}, \mu^{d}\right\rangle}\right\rangle_{\omega_{b}, \omega_{c}, \omega_{d}}\left(\bar{x}^{c d}\right)\right. \\
& \approx-\sum_{q>0} \frac{\Phi_{q}^{a b c d}(\omega)+\Lambda_{q}^{a b c d}(\omega)}{\omega_{q}-2 \omega-i \gamma} \\
& =-\sum_{q>0}\left[\Phi_{q}^{a b c d}(\omega)+\Lambda_{q}^{a b c d}(\omega)\right]\left[\mathcal{D}_{q}(2 \omega)+i \mathcal{A}_{q}(2 \omega)\right]
\end{aligned}
$$

Before proceeding let us consider the damped linear response function $\left\langle\left\langle\overline{\mu^{a} ; \mu^{b}}\right\rangle\right\rangle_{\omega}$ which is the one-photon analogue of the damped TPA response function in Eq. (46). The damped linear response function is given by (see, e.g., Ref. 8)

$$
\left\langle\left\langle\overline{\mu^{a} ; \mu^{b}}\right\rangle\right\rangle_{\omega} \approx-\sum_{q>0} \mu_{0 q}^{a} \mu_{q 0}^{b}\left[\mathcal{D}_{q}(\omega)+i \mathcal{A}_{q}(\omega)\right] .
$$

The imaginary part of the damped linear response function represents a spectrum of the $(a, b)$ th component of the $O P A$ tensor $\left[\mu_{0 q}^{a} \mu_{q 0}^{b}\right]$ with superimposed Lorentzian lineshape functions $\mathcal{A}_{q}(\omega)$, whereas the real part describes a dispersion spectrum. Noticing the similarities between Eqs. (46) and (47) we, therefore, define a damped TPA function $\delta_{D}^{a b c d}(\omega)$, which equals minus the imaginary part of Eq. (46),

$$
\begin{aligned}
\delta_{D}^{a b c d}(\omega) & \equiv-\operatorname{Im}\left[\left\langle\left\langle\overline{\mu^{a} ; \mu^{b}, \mu^{c}, \mu^{d}}\right\rangle\right\rangle_{\omega_{b}, \omega_{c}, \omega_{d}}\left(\bar{x}^{c d}\right)\right] \\
& \approx \delta_{\Phi}^{a b c d}(\omega)+\delta_{\Lambda}^{a b c d}(\omega)
\end{aligned}
$$

where

$$
\begin{aligned}
& \delta_{\Phi}^{a b c d}(\omega)=\sum_{q>0} \Phi_{q}^{a b c d}(\omega) \mathcal{A}_{q}(2 \omega) \\
& \delta_{\Lambda}^{a b c d}(\omega)=\sum_{q>0} \Lambda_{q}^{a b c d}(\omega) \mathcal{A}_{q}(2 \omega) .
\end{aligned}
$$

The isotropically averaged damped TPA spectrum $\bar{\delta}_{D}(\omega)$ is obtained as in Eq. (5)

$$
\bar{\delta}_{D}(\omega)=\frac{1}{30} \sum_{a, b}\left\{F \delta_{D}^{a a b b}(\omega)+(G+H) \delta_{D}^{a b a b}(\omega)\right\} .
$$

We note that the imaginary part of the damped linear response function in Eq. (47) is identical to a one-photon stick spectrum with superimposed lineshape functions. The damped TPA function $\bar{\delta}_{D}(\omega)$ is in general not completely identical to the standard TPA strengths in Eq. (4) with superimposed lineshape functions. However, in Sec. III A we show that in the single-resonance case [Fig. 1(a)] the damped and standard TPA spectra are - for all practical purposes identical. In contrast, in the double-resonance case depicted in Fig. 1(b), the standard TPA spectrum diverges, whereas the damped TPA spectrum is still well-defined and physically meaningful. We elaborate on this matter in Sec. III B.

\section{COMPARISON OF STANDARD AND DAMPED TPA}

In this section we compare standard and damped TPA spectra in the single (Sec. III A and double (Sec. III B) resonance cases, corresponding to Figs. 1(a) and 1(b), respectively. In Sec. III C, we also compare standard and damped TPA spectra over a broader frequency range. The discussions are accompanied by illustrative TPA spectra for small test systems calculated using DFT or HF response theory. We postpone the implementation details to Sec. IV and focus here only on the physical interpretation of the damped TPA spectra.

\section{A. The single-resonance case}

The broadening factor $\gamma$ is in general much smaller than the optical frequencies, so if the optical frequency in Eq. (30) is far from $\omega_{k}$ we may omit the $\gamma^{2}$ factor in the denominator of $\mathcal{D}_{k}(\omega)$, whereas $\mathcal{A}_{k}(\omega)$ is close to zero:

$$
\left.\mathcal{D}_{k}(\omega) \approx \frac{1}{\omega_{k}-\omega} ; \quad \mathcal{A}_{k}(\omega) \approx 0 \quad \text { ( } \omega \text { far-off-resonance }\right)
$$

Thus, for analysis purposes, the replacement in Eq. (29) is important only when the frequency in question is close to an excitation energy.

Applying the approximation in Eq. (51) we see that $\Lambda_{q}^{a b c d}(\omega)$ in Eq. (45b) is close to zero,

$$
\Lambda_{q}^{a b c d}(\omega) \approx 0 \quad(\omega \text { far-off-resonance }),
$$

whereas, using Eqs. (3) and (45a), $\Phi_{q}^{a b c d}(\omega)$ approximately equals the standard TPA strength evaluated at the optical 


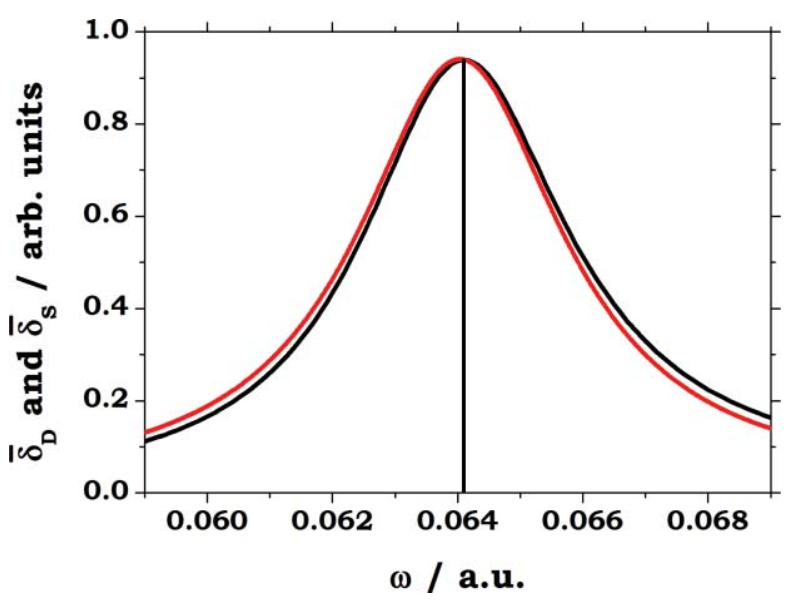

FIG. 2. Standard (red) and damped (black) TPA spectra for LiH at the CAMB3LYP/aug-cc-pVTZ level of theory using the experimental equilibrium geometry $1.5957 \AA$ (Ref. 97). The broadening parameter $\gamma=0.004$ a.u. Note that the optical frequency $\omega$ corresponds to a one-photon frequency.

frequency $\omega$,

$$
\Phi_{q}^{a b c d}(\omega) \approx T_{0 q}^{a b}(\omega, \omega) T_{0 q}^{c d}(\omega, \omega) \quad(\omega \text { far-off-resonance }) .
$$

Inserting Eq. (53) into Eq. (49a) the damped TPA function may be written as

$$
\delta_{D}^{a b c d}(\omega) \approx \delta_{\Phi}^{a b c d}(\omega) \approx \sum_{q>0} T_{0 q}^{a b}(\omega, \omega) T_{0 q}^{c d}(\omega, \omega) \mathcal{A}_{q}(2 \omega)
$$

( $\omega$ far-off-resonance).

This expression is very similar but not identical to the corresponding standard TPA residue spectrum with superimposed lineshape functions $\delta_{S}^{a b c d}(\omega)$, which, using Eq. (27), has the form

$$
\delta_{S}^{a b c d}(\omega)=\sum_{q>0} T_{0 q}^{a b}\left(\omega_{q} / 2, \omega_{q} / 2\right) T_{0 q}^{c d}\left(\omega_{q} / 2, \omega_{q} / 2\right) \mathcal{A}_{q}(2 \omega) .
$$

The TPA amplitudes in the damped TPA spectrum in Eq. (54) depend on the optical frequency $\omega$, whereas the frequencies entering the standard TPA amplitudes in Eq. (55) equal exactly half the excitation energies $\omega_{q} / 2$. In the singleresonance case the damped and standard TPA spectra will thus be very similar, but not identical.

To illustrate the discussion above we have, in Fig. 2, plotted the isotropically averaged damped TPA spectrum in Eq. (50) and the corresponding standard TPA spectrum $\bar{\delta}_{S}(\omega)$,

$$
\bar{\delta}_{S}(\omega)=\frac{1}{30} \sum_{a, b}\left\{F \delta_{S}^{a a b b}(\omega)+(G+H) \delta_{S}^{a b a b}(\omega)\right\},
$$

for a CAM-B3LYP (Ref. 80-82) calculation on the $\mathrm{LiH}$ molecule using the aug-cc-pVTZ basis. ${ }^{83}$ The first excited state in $\mathrm{LiH}\left(\omega_{1}=0.128\right.$ a.u. $)$ is relatively isolated, and therefore the $|0\rangle \rightarrow|1\rangle$ transition is appropriate to illustrate

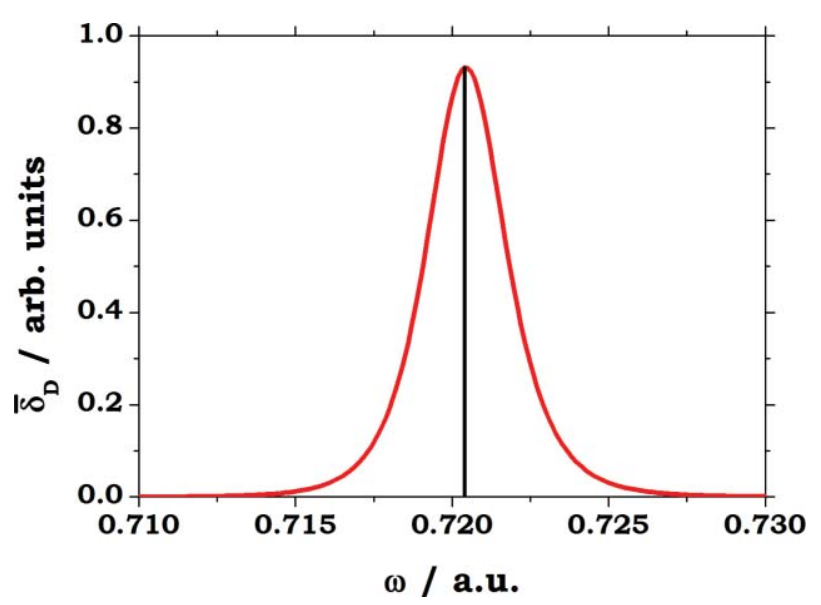

FIG. 3. Damped absorption spectra for the artificial hydrogen fluoride test system described in the text, where the third excitation energy $\omega_{3}$ equals half the fourth excitation energy $\omega_{4} / 2$ (indicated by black stick). The broadening parameter $\gamma=0.004$ a.u. Note that the optical frequency $\omega$ corresponds to a one-photon frequency.

the single-resonance case. The frequency range in Fig. 2 is therefore centered around $\omega_{1} / 2$ to probe two-photon excitation to $|1\rangle$. Clearly, for all practical purposes the standard and damped spectra are identical, but a closer inspection reveals that they differ slightly for the reasons discussed above.

\section{B. The double-resonance case}

We now consider the double-resonance case, where $\omega$ and $2 \omega$ are both close to resonance in the sense that $\omega \approx \omega_{m}$ and $2 \omega \approx \omega_{n}$, see Fig. 1(b). In this case the standard expression for the TPA amplitude in Eq. (4) becomes artificially large, and it diverges in the limit where $\omega=\omega_{m}=\omega_{n} / 2$. In damped response theory the singularity problems of standard response theory are effectively avoided by introducing a factor $i \gamma$ in the denominator of the standard TPA amplitudes, see, e.g., Eqs. (34) and (36). We now discuss the implications of this empirical solution to the singularity problem in the double-resonance case.

When $\omega$ is close to $\omega_{m}$ the $m$ th term will dominate the sum-over-states expression for the TPA amplitude in Eq. (4), and the expression for the standard TPA strength in Eq. (27) approximately becomes,

$$
\begin{aligned}
& T_{0 n}^{a b}\left(\omega_{n} / 2, \omega_{n} / 2\right) T_{0 n}^{c d}\left(\omega_{n} / 2, \omega_{n} / 2\right) \\
& \quad \approx P_{a b} P_{c d}\left(\mu_{0 m}^{a} \mu_{m n}^{b} \mu_{0 m}^{c} \mu_{m n}^{d}\right) \frac{1}{\left(\omega_{m}-\omega_{n} / 2\right)^{2}} \\
& \left(\omega \approx \omega_{m} \approx \omega_{n} / 2\right),
\end{aligned}
$$

which clearly diverges if $\omega_{m}=\omega_{n} / 2$. The permutation operator was defined in Eq. (18).

Considering damped TPA, the $n$th term will dominate the sum-over-states expressions in Eq. (49) when $2 \omega \approx \omega_{n}$ :

$$
\begin{gathered}
\delta_{\Phi}^{a b c d}(\omega) \approx \Phi_{n}^{a b c d}(\omega) \mathcal{A}_{n}(2 \omega) \quad\left(2 \omega \approx \omega_{n}\right), \\
\delta_{\Lambda}^{a b c d}(\omega) \approx \Lambda_{n}^{a b c d}(\omega) \mathcal{A}_{n}(2 \omega) \quad\left(2 \omega \approx \omega_{n}\right) .
\end{gathered}
$$


Furthermore, since $\omega \approx \omega_{m}$ the $m$ th term in Eq. (45) will dominate the overall shapes of $\Phi_{n}^{a b c d}(\omega)$ and $\Lambda_{n}^{a b c d}(\omega)$,

$$
\begin{aligned}
& \Phi_{n}^{a b c d}(\omega) \approx P_{a b} P_{c d}\left(\mu_{0 m}^{a} \mu_{m n}^{b} \mu_{0 m}^{c} \mu_{m n}^{d}\right) \mathcal{D}_{m}(\omega)^{2} \\
& \left(\omega \approx \omega_{m} \approx \omega_{n} / 2\right), \\
& \Lambda_{n}^{a b c d}(\omega) \approx P_{a b} P_{c d}\left(\mu_{0 m}^{a} \mu_{m n}^{b} \mu_{0 m}^{c} \mu_{m n}^{d}\right) \mathcal{A}_{m}(\omega)^{2} \\
& \left(\omega \approx \omega_{m} \approx \omega_{n} / 2\right),
\end{aligned}
$$

and Eq. (58) may thus be written as

$$
\begin{aligned}
& \delta_{\Phi}^{a b c d}(\omega) \approx P_{a b} P_{c d}\left(\mu_{0 m}^{a} \mu_{m n}^{b} \mu_{0 m}^{c} \mu_{m n}^{d}\right) \mathcal{D}_{m}(\omega)^{2} \mathcal{A}_{n}(2 \omega) \\
& \left(\omega \approx \omega_{m} \approx \omega_{n} / 2\right) \\
& \delta_{\Lambda}^{a b c d}(\omega) \approx P_{a b} P_{c d}\left(\mu_{0 m}^{a} \mu_{m n}^{b} \mu_{0 m}^{c} \mu_{m n}^{d}\right) \mathcal{A}_{m}(\omega)^{2} \mathcal{A}_{n}(2 \omega) \\
& \left(\omega \approx \omega_{m} \approx \omega_{n} / 2\right) .
\end{aligned}
$$

A function analysis shows that in the approximate double-resonance case $\mathcal{A}_{m}(\omega)^{2} \mathcal{A}_{n}(2 \omega)$ completely dominates compared to $\mathcal{D}_{m}(\omega)^{2} \mathcal{A}_{n}(2 \omega)$. Therefore, the form of the TPA function $\delta_{D}^{a b c d}$ in Eq. (48) will be determined by $\delta_{\Lambda}^{a b c d}(\omega)$ and the $\delta_{\Phi}^{a b c d}(\omega)$ term may be neglected:

$$
\begin{aligned}
\delta_{D}^{a b c d}(\omega) \approx & \delta_{\Lambda}^{a b c d}(\omega) \approx P_{a b} P_{c d}\left(\mu_{0 m}^{a} \mu_{m n}^{b} \mu_{0 m}^{c} \mu_{m n}^{d}\right) \\
& \times \mathcal{A}_{m}(\omega)^{2} \mathcal{A}_{n}(2 \omega) \quad\left(\omega \approx \omega_{m} \approx \omega_{n} / 2\right) .
\end{aligned}
$$

This is to be contrasted with the single-resonance case in Eq. (54), where $\delta_{\Phi}^{a b c d}(\omega)$ is the dominant contribution.

To investigate in more detail the physical content of Eq. (61) let us consider the exact double-resonance case $\omega$ $=\omega_{m}=\omega_{n} / 2$, see Fig. 1(b), and assume that $a=b=c$ $=d=x$ (TPA absorption along the $x$ axis):

$$
\delta_{D}^{x x x x}(\omega) \approx 4\left|\mu_{0 m}^{x}\right|^{2}\left|\mu_{m n}^{x}\right|^{2}\left(8 \tau^{3}\right) \quad\left(\omega=\omega_{m}=\omega_{n} / 2\right),
$$

where we have used Eq. (30b) and inserted the empirical lifetime $\tau=(2 \gamma)^{-1}$. A simple physical interpretation of the TPA process described by Eq. (62) is the following:

(1) First, the one-photon excitation $|0\rangle \rightarrow|m\rangle$ occurs with transition strength $\left|\mu_{0 m}^{x}\right|^{2}$, corresponding to the first transition in Fig. 1(b). This process is proportional to the lifetime of $|m\rangle(\tau)$;

(2) Following the $|0\rangle \rightarrow|m\rangle$ transition, a second onephoton transition $|m\rangle \rightarrow|n\rangle$ occurs with transition strength $\left|\mu_{m n}^{x}\right|^{2}$ [the second transition in Fig. 1(b)]. This process is proportional to the lifetime of $|m\rangle(\tau)$ and to the lifetime of $|n\rangle$ (also $\tau$ because we simply assume that all excited states have the same lifetime).

The total TPA process is therefore proportional to $\left|\mu_{0 m}^{x}\right|^{2} \tau$ (first transition) times $\left|\mu_{m n}^{x}\right|^{2} \tau^{2}$ (second transition). This interpretation is also equivalent with what Sakurai ${ }^{2}$ obtains by using a slow turn-on method for the external potential. We emphasize, however, that no real information is contained in the lifetime $\tau$, because it merely is an empirical pa-

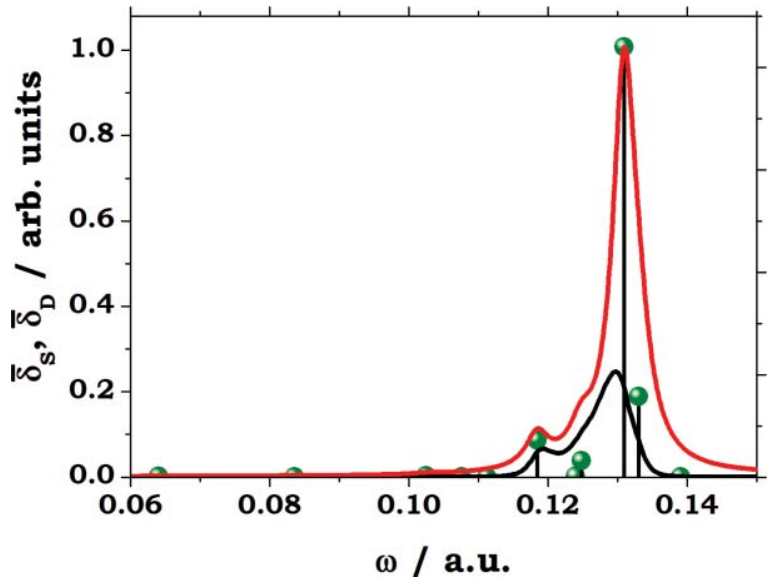

FIG. 4. Standard (red) and damped (black) TPA spectra for LiH at the CAMB3LYP/aug-cc-pVTZ level of theory using the experimental equilibrium geometry $1.5957 \AA$ (Ref. 97). The broadening parameter $\gamma=0.004$ a.u. Note that the optical frequency $\omega$ corresponds to a one-photon frequency.

rameter, and, therefore, the interpretation given above is only qualitative.

Let us now consider the exact double-resonance case discussed above using a small test calculation. The test system is hydrogen fluoride with an $H-F$ bond length of 1.95477 a.u., and with 6-31G basis functions on $F$ and an STO-3G basis function on $H$. We emphasize that this very artificial system is in no way a realistic representation of the hydrogen fluoride molecule; however, it serves as a useful test system, because the third and fourth excitation energies are related by $\omega_{3}=\omega_{4} / 2=0.7204$ a.u. at the Hartree-Fock $(\mathrm{HF})$ level of theory. This system, thus, illustrates the structure of damped TPA spectra in the general double-resonance case.

A standard TPA calculation of the $|0\rangle \rightarrow|4\rangle$ two-photon transition for this HF molecule will give meaninglessly high numbers, if the response equations converge at all. In contrast, the damped TPA calculation gives a smooth spectrum centered at $\omega=\omega_{3}=\omega_{4} / 2=0.7204$ a.u., see Fig. 3. The damped TPA formulation thus provides a meaningful spectrum, also in double-resonance cases.

\section{TPA spectra over a broad frequency range}

We have now discussed the single-resonance (Sec. III A) and double-resonance (Sec. III B) cases by zooming into single peaks in the TPA spectrum. A general TPA spectrum will contain a mixture of (approximate) single- and double-resonance transitions corresponding to excitations from the ground state to different excited states. Let us, therefore, also compare standard and damped TPA spectra in a broader frequency range. This is done in Fig. 4, where we have plotted standard (red) and damped (black) TPA spectra for a CAM-B3LYP/aug-cc-pVTZ calculation on the $\mathrm{LiH}$ molecule.

The two spectra in Fig. 4 have similar shapes, but their appearances are still quite different, because the excitation spectrum contains approximate double-resonances, which, as discussed in Sec. III B, cause the standard TPA spectrum to blow up compared to the damped spectrum. For 
example, the one-photon frequency for the largest peak ( $\omega \approx 0.131$ a.u.) is approximately twice that of the lowest excitation ( $\omega \approx 0.064$ a.u.). Thus, for the approximate doubleresonance at $\omega \approx 0.131$ a.u., the standard TPA values are much larger than the corresponding damped values. The validity of Eq. (4) is therefore highly questionable in this case. Note also that in both standard and damped spectra, approximate double-resonance peaks completely dominate the spectra compared to single-resonance peaks.

Summarizing, in the single-resonance case the standard and damped TPA spectra are - for all practical purposes identical, whereas the occurrence of a double-resonance causes the standard TPA spectrum to blow up. In contrast, the damped TPA spectrum is well-defined and provides physically motivated TPA spectra at all optical frequencies. It should also be kept in mind that the exact appearance of the damped TPA spectrum depends on the empirical broadening parameter $\gamma$, which determines the width of the absorption peaks.

\section{TWO-PHOTON ABSORPTION IN DENSITY FUNCTIONAL THEORY}

In this section we describe how standard and damped TPA spectra may be evaluated using the atomic orbital (AO) based DFT response formulation developed in Ref. 84. Our aim is to develop DFT expressions, which resemble the expressions in exact theory presented in Sec. II as closely as possible. In Sec. IV A, we carry out a residue analysis of the standard TPA response function $\left\langle\left\langle\mu^{a} ; \mu^{b}, \mu^{c}, \mu^{d}\right\rangle\right\rangle_{\omega_{b}, \omega_{c}, \omega_{d}}\left(X^{c d}\right)$ to obtain a standard TPA stick spectrum; and in Sec. IV B, we introduce damping terms into the standard TPA response function to obtain a damped TPA response function $\left\langle\left\langle\overline{\mu^{a} ; \mu^{b}, \mu^{c}, \mu^{d}}\right\rangle\right\rangle_{\omega_{b}, \omega_{c}, \omega_{d}}\left(\bar{X}^{c d}\right)$.

\section{A. Standard two-photon absorption}

In Sec. II A, we demonstrated that the standard TPA response function may be written in the form of Eq. (23) for any variational electron structure model including KS-DFT. The density matrix-based DFT analogue of Eq. (23) becomes,

$$
\left\langle\left\langle\mu^{a} ; \mu^{b}, \mu^{c}, \mu^{d}\right\rangle\right\rangle_{\omega_{b}, \omega_{c}, \omega_{d}}\left(X^{c d}\right)=\operatorname{Tr}^{a b} \mathbf{X}^{c d},
$$

where $\mathbf{g}^{a b}$ is the second-order right-hand side matrix and $\mathbf{X}^{c d}$ is the second-order response matrix. We mention that we have also explicitly derived Eq. (63) based on the formulation in Ref. 84, but the derivation is quite lengthy and we shall refrain from presenting it here. The right-hand side matrix $\mathbf{g}^{a b}$ is given by

$$
\begin{aligned}
\mathbf{g}^{a b}= & \mathcal{P}^{\mathrm{ov}}\left[\mathbf{F}_{1}^{a b}\left(\mathbf{D}^{a}, \mathbf{D}^{b}, \mathbf{D}_{P}^{a b}\right) \mathbf{D S}-\mathbf{S D F}_{1}^{a b}\left(\mathbf{D}^{a}, \mathbf{D}^{b}, \mathbf{D}_{P}^{a b}\right)\right. \\
& \left.+P_{a b}\left\{\mathbf{F}^{a}\left(\mathbf{D}^{a}\right) \mathbf{D}^{b} \mathbf{S}-\mathbf{S D}^{b} \mathbf{F}^{a}\left(\mathbf{D}^{a}\right)\right\}\right],
\end{aligned}
$$

where $\mathbf{D}$ is the optimized AO density matrix for the unperturbed molecule and $\mathbf{S}$ is the AO overlap matrix,

$$
S_{\mu \nu}=\left\langle\chi_{\mu} \mid \chi_{\nu}\right\rangle
$$

The $\mathcal{P}^{\text {ov }}$ operator projects out the occupied-virtual and virtual-occupied components of a matrix $\mathbf{A}$

$$
\mathcal{P}^{\mathrm{ov}} \mathbf{A}=\mathbf{P}^{T} \mathbf{A} \mathbf{Q}+\mathbf{Q}^{T} \mathbf{A} \mathbf{P},
$$

where the $\mathbf{P}$ and $\mathbf{Q}$ matrices are projectors onto the occupied and virtual spaces, respectively,

$$
\begin{gathered}
\mathbf{P}=\mathbf{D S}, \\
\mathbf{Q}=\mathbf{1}-\mathbf{P}=\mathbf{1}-\mathbf{D S} .
\end{gathered}
$$

We note that Eq. (64) corresponds to Eq. (187) in Ref. 84, where the terms associated with perturbation-dependent basis sets (which are not used in this work) have been omitted. In addition, the explicit use of the projection operator $\mathcal{P}^{\text {ov }}$ in Eq. (64) has allowed us to simplify Eq. (187) in Ref. 84 further by removing some redundant terms which do not comply with the projection properties of $\mathbf{g}^{a b}$ :

$$
\mathbf{g}^{a b}=\mathcal{P}^{\mathrm{ov}} \mathbf{g}^{a b} .
$$

The first-order derivative of the density matrix with respect to perturbation $b$ (in the frequency domain) is denoted by $\mathbf{D}^{b}$ and may be written in the following form ${ }^{84}$

$$
\mathbf{D}^{b}=\mathbf{D S X}^{b}-\mathbf{X}^{b} \mathbf{S D},
$$

where $\mathbf{X}^{b}$ is the first-order response matrix for the $\mu^{b}$ perturbation which is obtained by solving the first-order response equation

$$
\left(\mathbf{E}^{[2]}-\omega_{b} \mathbf{S}^{[2]}\right) \mathbf{X}^{b}=\mathbf{g}^{b} .
$$

The first-order right-hand side matrix $\mathbf{g}^{b}$ is given by

$$
\mathbf{g}^{b}=\boldsymbol{\mu}^{b} \mathbf{D S}-\mathbf{S D} \boldsymbol{\mu}^{b},
$$

where

$$
\mu_{\kappa \lambda}^{b}=\left\langle\chi_{\kappa}\left|\mu^{b}\right| \chi_{\lambda}\right\rangle .
$$

Equations (71) and (72) are the DFT equivalents of the exact expressions in Eqs. (15a) and (17), respectively. We refer to Ref. 84 for the definitions of the linear transformations $\mathbf{E}^{[2]} \mathbf{X}^{b}$ and $\mathbf{S}^{[2]} \mathbf{X}^{b}$ in AO-based DFT.

The $\mathbf{F}^{a}\left(\mathbf{D}^{a}\right)$ matrix in Eq. (64) is the total first-order derivative of the Kohn-Sham(KS) matrix with respect to perturbation $a$ (in the frequency domain),

$$
\mathbf{F}^{a}\left(\mathbf{D}^{a}\right)=\mathbf{G}^{K S}\left(\mathbf{D}^{a}\right)+\boldsymbol{\mu}^{a},
$$

where the two-electron KS matrix $\mathbf{G}^{K S}\left(\mathbf{D}^{a}\right)$ is a sum of the two-electron matrix with $\eta$-scaled exchange $\mathbf{G}^{\eta}\left(\mathbf{D}^{a}\right)$ and the exchange-correlation matrix $\mathbf{G}^{x c}\left(\mathbf{D}^{a}\right)$,

$$
\mathbf{G}^{K S}\left(\mathbf{D}^{a}\right)=\mathbf{G}^{\eta}\left(\mathbf{D}^{a}\right)+\mathbf{G}^{x c}\left(\mathbf{D}^{a}\right),
$$

$$
G_{\kappa \lambda}^{\eta}\left(\mathbf{D}^{a}\right)=\sum_{\alpha \beta} D_{\beta \alpha}^{a}\left(g_{\kappa \lambda \alpha \beta}-\eta g_{\kappa \beta \alpha \lambda}\right),
$$

$$
G_{\kappa \lambda}^{x c}\left(\mathbf{D}^{a}\right)=\sum_{\alpha \beta} D_{\beta \alpha}^{a} \int \Omega_{\kappa \lambda}(\mathbf{r})\left(\int \Omega_{\alpha \beta}\left(\mathbf{r}_{1}\right) \frac{\delta v_{x c}\left(\mathbf{r}, \mathbf{r}_{1}\right)}{\delta \rho\left(\mathbf{r}_{1}\right)} d \mathbf{x}_{1}\right) d \mathbf{x}
$$


where $\boldsymbol{\Omega}$ is the overlap distribution,

$$
\Omega_{\kappa \lambda}\left(\mathbf{r}_{1}\right)=\chi_{\kappa}\left(\mathbf{r}_{1}\right) \chi_{\lambda}\left(\mathbf{r}_{1}\right)
$$

$v_{x c}$ is the exchange-correlation potential and $\rho\left(\mathbf{r}_{1}\right)$ is the electron density at the position $\mathbf{r}_{1}$. The integration variable $\mathbf{x}$ (not to be confused with the response parameters) refers to both spin and spatial components, ${ }^{84}$ and the two-electron integrals are given by

$$
g_{\kappa \lambda \alpha \beta}=\iint \chi_{\kappa}^{*}\left(\mathbf{x}_{1}\right) \chi_{\lambda}\left(\mathbf{x}_{1}\right) \frac{1}{r_{12}} \chi_{\alpha}^{*}\left(\mathbf{x}_{2}\right) \chi_{\beta}\left(\mathbf{x}_{2}\right) d \mathbf{x}_{1} d \mathbf{x}_{2} .
$$

The $\mathbf{F}_{1}^{a b}\left(\mathbf{D}^{a}, \mathbf{D}^{b}, \mathbf{D}_{P}^{a b}\right)$ matrix in Eq. (64) is the secondorder derivative of the KS matrix with respect to perturbations $a$ and $b$ (in the frequency domain), where only the terms containing first-order response parameters $\left(X^{a}\right.$ and $\left.X^{b}\right)$ have been kept (hence the " 1 " subscript), ${ }^{84}$

$$
\left[F_{1}^{a b}\left(\mathbf{D}^{a}, \mathbf{D}^{b}, \mathbf{D}_{P}^{a b}\right)\right]_{\kappa \lambda}=\mathbf{G}^{K S}\left(\mathbf{D}_{P}^{a b}\right)+\int \Omega_{\kappa \lambda}(\mathbf{r}) v_{x c, 1}^{a b}(\mathbf{r}) d \mathbf{r},
$$

where we have introduced the particular component of the second-order density matrix, ${ }^{84}$ which contains only first-order response parameters,

$$
\mathbf{D}_{P}^{a b}=\mathbf{P} \mathbf{K}^{a b} \mathbf{P}^{T}-\mathbf{Q} \mathbf{K}^{a b} \mathbf{Q}^{T}
$$

with

$$
\mathbf{K}^{a b}=-\mathbf{D}^{a} \mathbf{S D}^{b}-\mathbf{D}^{b} \mathbf{S D}^{a} .
$$

The second order derivative of the exchange-correlation potential $v_{x c, 1}^{a b}$ with respect to perturbations $a$ and $b$ (leaving out second-order response parameters) may be written in the form $^{84}$

$$
\begin{aligned}
v_{x c, 1}^{a b}= & \int \frac{\delta v_{x c}\left(\mathbf{r}, \mathbf{r}_{1}\right)}{\delta \rho\left(\mathbf{r}_{1}\right)} \rho_{1}^{a b}\left(\mathbf{r}_{1}\right) d \mathbf{x}_{1} \\
& +\iint \frac{\delta^{2} v_{x c}\left(\mathbf{r}, \mathbf{r}_{1}, \mathbf{r}_{2}\right)}{\delta \rho\left(\mathbf{r}_{1}\right) \delta \rho\left(\mathbf{r}_{2}\right)} \rho^{a}\left(\mathbf{r}_{1}\right) \rho^{b}\left(\mathbf{r}_{2}\right) d \mathbf{x}_{1} d \mathbf{x}_{2},
\end{aligned}
$$

where the first- and second-order perturbed electron densities (leaving out second-order response parameters) are given by

$$
\begin{gathered}
\rho^{a}=\operatorname{Tr} \boldsymbol{\Omega} \mathbf{D}^{a}, \\
\rho^{b}=\operatorname{Tr} \boldsymbol{\Omega} \mathbf{D}^{b}, \\
\rho_{1}^{a b}=\operatorname{Tr} \boldsymbol{\Omega} \mathbf{D}_{P}^{a b} .
\end{gathered}
$$

The second-order response matrix $\mathbf{X}^{c d}$ in Eq. (63) contains only occupied-virtual and virtual-occupied components, ${ }^{84}$ i.e.,

$$
\mathbf{X}^{c d}=\mathcal{P}^{\text {ov }} \mathbf{X}^{c d}
$$

and is obtained by solving the second-order response equation $^{84}$

$$
\left(\mathbf{E}^{[2]}-\omega_{c d} \mathbf{S}^{[2]}\right) \mathbf{X}^{c d}=\mathbf{g}^{c d}
$$

where $\mathbf{g}^{c d}$ is given by Eq. (64) with $a \rightarrow c$ and $b \rightarrow d$.

From the frequency relations in Eq. (26) it is clear that to determine $\mathbf{X}^{c}\left(\mathbf{X}^{d}\right)$, Eq. (71) should be solved for a positive optical frequency $\omega_{c}=\omega\left(\omega_{d}=\omega\right)$, whereas the determination of $\mathbf{X}^{a}\left(\mathbf{X}^{b}\right)$ requires solving Eq. (71) for a negative optical frequency $\omega_{a}=-\omega\left(\omega_{b}=-\omega\right)$. However, due to the pairing properties of the $\mathbf{E}^{[2]}$ and $\mathbf{S}^{[2]}$ matrices, see, e.g., Ref. $85, \mathbf{X}^{a}(-\omega)$ and $\mathbf{X}^{c}(\omega)$ are related in a simple manner (for $a=c$ ),

$$
\mathbf{X}^{a}(-\omega)=-\left[\mathbf{X}^{c}(\omega)\right]^{T} \quad(a=c),
$$

where we have added frequency arguments for clarity. The relation in Eq. (89) implies the following symmetry relation between the second-order right-hand side matrices:

$$
\mathbf{g}^{a b}(-\omega,-\omega)=-\left[\mathbf{g}^{c d}(\omega, \omega)\right]^{T} \quad(a=c ; \quad b=d),
$$

which will prove useful for the analysis below. In Eq. (90) and in the following analysis we have included frequency arguments in the right-hand side matrices for clarity.

Let us now determine the residue of $\left\langle\left\langle\mu^{a} ; \mu^{b}, \mu^{c}, \mu^{d}\right\rangle\right\rangle_{\omega_{b}, \omega_{c}, \omega_{d}}\left(X^{c d}\right)$ in Eq. (63) at $\omega_{c d}=\omega_{n}$, which determines the TPA strength for the $|0\rangle \rightarrow|n\rangle$ transition. This is the DFT analogue of the exact residue expression in Eq. (27). It is convenient first to rewrite Eq. (63) using the supermatrix notation, see, e.g., Refs. 8, 85, and 86, where the AO indices $\mu \nu$ (Greek letters) are collected in one index $I$ (Roman letter), e.g,

$$
X_{\mu \nu}^{c d} \rightarrow X_{I} ; \quad E_{\mu \nu \rho \sigma}^{[2]} \rightarrow E_{I J}^{[2]}
$$

With this supermatrix notation a two-index matrix $\left(\mathbf{X}^{c d}\right)$ is written in bold Roman font, whereas its vector analogue $\left(\mathbf{X}^{c d}\right)$ is written in bold italic font.

Using Eq. (90) and the supermatrix notation introduced above we may rewrite Eq. (63) in a form, which is more useful for determining the TPA residue,

$$
\begin{aligned}
\left\langle\left\langle\mu^{a} ; \mu^{b}, \mu^{c}, \mu^{d}\right\rangle\right\rangle_{\omega_{b}, \omega_{c}, \omega_{d}}\left(X^{c d}\right) & =\operatorname{Trg}^{a b}(-\omega,-\omega) \mathbf{X}^{c d} \\
& =\sum_{\mu \nu} g_{\nu \mu}^{a b}(-\omega,-\omega) X_{\mu \nu}^{c d} \\
& =-\sum_{\mu \nu} g_{\mu \nu}^{a b}(\omega, \omega) X_{\mu \nu}^{c d} \\
& =-\sum_{I} g_{I}^{a b}(\omega, \omega) X_{I}^{c d}
\end{aligned}
$$

To be able to identify the residue of Eq. (92) let us rewrite Eq. (88) in the supermatrix notation,

$$
\boldsymbol{X}^{c d}=\left(\boldsymbol{E}^{[2]}-\omega_{c d} \boldsymbol{S}^{[2]}\right)^{-1} \boldsymbol{g}^{c d}
$$


and introduce the spectral representation of $\left(E^{[2]}\right.$ $\left.-\omega_{c d} S^{[2]}\right)^{-1}($ Ref. 86)

$$
\begin{aligned}
\left(\boldsymbol{E}^{[2]}-\omega_{c d} \boldsymbol{S}^{[2]}\right)^{-1} & =\sum_{p>0}\left[\left(\omega_{p}-\omega_{c d}\right)^{-1} \boldsymbol{X}_{p} \boldsymbol{X}_{p}^{T}\right. \\
& \left.+\left(\omega_{p}+\omega_{c d}\right)^{-1} \boldsymbol{X}_{-p} \boldsymbol{X}_{-p}^{T}\right]
\end{aligned}
$$

where $\omega_{p}$ is the $p$ th excitation energy and $\boldsymbol{X}_{p}$ is an eigenvector obtained from the generalized eigenvalue problem

$$
\left(\boldsymbol{E}^{[2]}-\omega_{p} \boldsymbol{S}^{[2]}\right) \boldsymbol{X}_{p}=\mathbf{0} .
$$

Using Eqs. (93) and (94) we see that

$$
\lim _{\omega_{c d} \rightarrow \omega_{n}}\left(\omega_{c d}-\omega_{n}\right) \boldsymbol{X}^{c d}=-\boldsymbol{X}_{n}\left(\boldsymbol{X}_{n}^{T} \boldsymbol{g}^{c d}\right) .
$$

Note that $\left(\boldsymbol{X}_{n}^{T} \boldsymbol{g}^{c d}\right)$ is just a number. By inserting Eq. (96) into Eq. (92) we may now identify the residue of the cubic response function at $\omega_{c d}=\omega_{n}$,

$$
\begin{aligned}
\lim _{\omega_{c d} \rightarrow \omega_{n}} & \left(\omega_{c d}-\omega_{n}\right)\left\langle\left\langle\mu^{a} ; \mu^{b}, \mu^{c}, \mu^{d}\right\rangle\right\rangle_{\omega_{b}, \omega_{c}, \omega_{d}} \\
& =\lim _{\omega_{c d} \rightarrow \omega_{n}}\left(\omega_{c d}-\omega_{n}\right)\left\langle\left\langle\mu^{a} ; \mu^{b}, \mu^{c}, \mu^{d}\right\rangle\right\rangle_{\omega_{b}, \omega_{c}, \omega_{d}}\left(X^{c d}\right) \\
& =\left[\boldsymbol{g}^{a b}\left(\omega_{n} / 2, \omega_{n} / 2\right)^{T} \boldsymbol{X}_{n}\right]\left[\boldsymbol{X}_{n}^{T} \boldsymbol{g}^{c d}\left(\omega_{n} / 2, \omega_{n} / 2\right)\right] \\
& =T_{0 n}^{a b}\left(\omega_{n} / 2, \omega_{n} / 2\right) T_{0 n}^{c d}\left(\omega_{n} / 2, \omega_{n} / 2\right)
\end{aligned}
$$

where we have made the identifications,

$$
\begin{aligned}
& T_{0 n}^{a b}\left(\omega_{n} / 2, \omega_{n} / 2\right)=\sum_{I} g_{I}^{a b}\left(\omega_{n} / 2, \omega_{n} / 2\right)\left(X_{n}\right)_{I}, \\
& T_{0 n}^{c d}\left(\omega_{n} / 2, \omega_{n} / 2\right)=\sum_{I} g_{I}^{c d}\left(\omega_{n} / 2, \omega_{n} / 2\right)\left(X_{n}\right)_{I},
\end{aligned}
$$

to obtain TPA amplitudes in DFT, which are analogous to those in exact theory, compare Eqs. (97) and (98) to Eqs. (27) and (20). We note that the corresponding HF expressions are obtained if we set $\eta=1$ in Eq. (76) and omit all exchange-correlation terms.

\section{B. Damped two-photon absorption}

In this section we introduce complex excitation energies according to Eq. (29) into the standard TPA response function in Eq. (63) to obtain a damped TPA response function,

$$
\left\langle\left\langle\overline{\mu^{a} ; \mu^{b}, \mu^{c}, \mu^{d}}\right\rangle\right\rangle_{\omega_{b}, \omega_{c}, \omega_{d}}\left(\bar{X}^{c d}\right)=\operatorname{Tr} \overline{\mathbf{g}}^{a b} \overline{\mathbf{X}}^{c d},
$$

where a bar indicates that the replacement in Eq. (29) has been carried out. Equation (99) is the DFT analogue of the exact damped expression in Eq. (38), and to understand its structure we need to consider how the replacement in Eq. (29) is carried out in DFT.

Introducing the replacements in Eq. (29) the $\left(E^{[2]}\right.$ $\left.-\omega_{\alpha} S^{[2]}\right)^{-1}$ matrix in Eq. (94) may be written in the follow- ing way for a general frequency $\omega_{\alpha}:{ }^{8}$

$$
\begin{aligned}
\left(\boldsymbol{E}^{[2]}-\omega_{\alpha} \boldsymbol{S}^{[2]}\right)^{-1}= & \sum_{p>0}\left[\left(\omega_{p}-\omega_{\alpha}\right)^{-1} \boldsymbol{X}_{p} \boldsymbol{X}_{p}^{T}\right. \\
& \left.+\left(\omega_{p}+\omega_{\alpha}\right)^{-1} \boldsymbol{X}_{-p} \boldsymbol{X}_{-p}^{T}\right] \\
\rightarrow & \sum_{p>0}\left[\left(\omega_{p}-\omega_{\alpha}-i \gamma\right)^{-1} \boldsymbol{X}_{p} \boldsymbol{X}_{p}^{T}\right. \\
& \left.+\left(\omega_{p}+\omega_{\alpha}+i \gamma\right)^{-1} \boldsymbol{X}_{-p} \boldsymbol{X}_{-p}^{T}\right] \\
= & {\left[\boldsymbol{E}^{[2]}-\left(\omega_{\alpha}+i \gamma\right) \boldsymbol{S}^{[2]}\right]^{-1} . }
\end{aligned}
$$

The replacement in Eq. (100) transforms the standard DFT equations in Eqs. (71) and (88) into their damped counterparts,

$$
\begin{gathered}
{\left[\mathbf{E}^{[2]}-\left(\omega_{b}+i \gamma\right) \mathbf{S}^{[2]}\right] \overline{\mathbf{X}}^{b}=\mathbf{g}^{b},} \\
{\left[\mathbf{E}^{[2]}-\left(\omega_{c d}+i \gamma\right) \mathbf{S}^{[2]}\right] \overline{\mathbf{X}}^{c d}=\overline{\mathbf{g}}^{c d} .}
\end{gathered}
$$

Equation (101) is the DFT equivalent of the exact response equations in Eq. (31). Note that the first-order right-hand side matrix $\mathbf{g}^{b}$ is real and given by Eq. (72), whereas the secondorder right-hand side matrix $\overline{\mathbf{g}}^{c d}$ is a complex quantity.

The second-order right-hand side matrix $\overline{\mathbf{g}}^{a b}$ is obtained by introducing complex excitation energies into Eq. (64) by means of Eq. (100), i.e., the standard response matrices $\mathbf{X}^{a}$ and $\mathbf{X}^{b}$ in Eq. (64) are replaced by their damped counterparts $\overline{\mathbf{X}}^{a}$ and $\overline{\mathbf{X}}^{b}$. Considering Eq. (70), this is equivalent to introducing damped perturbed density matrices according to

$$
\overline{\mathbf{D}}^{b}=\mathbf{D S} \overline{\mathbf{X}}^{b}-\overline{\mathbf{X}}^{b} \mathbf{S D} .
$$

Similarly, the damped second-order particular density matrix is obtained by replacing the standard matrices in Eqs. (81) and (82) by their damped counterparts,

$$
\overline{\mathbf{D}}_{P}^{a b}=\mathbf{P} \overline{\mathbf{K}}^{a b} \mathbf{P}^{T}-\mathbf{Q} \overline{\mathbf{K}}^{a b} \mathbf{Q}^{T},
$$

where

$$
\overline{\mathbf{K}}^{a b}=-\overline{\mathbf{D}}^{a} \mathbf{S} \overline{\mathbf{D}}^{b}-\overline{\mathbf{D}}^{b} \mathbf{S} \overline{\mathbf{D}}^{a} .
$$

Introducing the damped density matrices above into the standard second-order right-hand side matrix in Eq. (64), we obtain

$$
\begin{aligned}
\overline{\mathbf{g}}^{a b}= & \mathcal{P}^{\mathrm{ov}}\left[\mathbf{F}_{1}^{a b}\left(\overline{\mathbf{D}}^{a}, \overline{\mathbf{D}}^{b}, \overline{\mathbf{D}}_{P}^{a b}\right) \mathbf{D S}-\mathbf{S D F}_{1}^{a b}\left(\overline{\mathbf{D}}^{a}, \overline{\mathbf{D}}^{b}, \overline{\mathbf{D}}_{P}^{a b}\right)\right. \\
& \left.+P_{a b}\left\{\mathbf{F}^{a}\left(\overline{\mathbf{D}}^{a}\right) \overline{\mathbf{D}}^{b} \mathbf{S}-\mathbf{S} \overline{\mathbf{D}}^{b} \mathbf{F}^{a}\left(\overline{\mathbf{D}}^{a}\right)\right\}\right]
\end{aligned}
$$

and similarly for $\overline{\mathbf{g}}^{\text {cd }}$.

In Eq. (105), $\mathbf{F}^{a}\left(\overline{\mathbf{D}}^{a}\right)$ and $\mathbf{F}_{1}^{a b}\left(\overline{\mathbf{D}}^{a}, \overline{\mathbf{D}}^{b}, \overline{\mathbf{D}}_{P}^{a b}\right)$ are evaluated as in Eqs. (74) and (80), where the standard perturbed density matrices $\mathbf{D}^{a}, \mathbf{D}^{b}$, and $\mathbf{D}_{P}^{a b}$ have been replaced by their damped counterparts $\overline{\mathbf{D}}^{a}, \overline{\mathbf{D}}^{b}$, and $\overline{\mathbf{D}}_{P}^{a b}$.

In analogy with the standard symmetry relations for the first-order response parameters in Eq. (89) we have the 
following symmetry relation for the damped response matrices:

$$
\overline{\mathbf{X}}^{a}(-\omega+i \gamma)=-\left[\overline{\mathbf{X}}^{c}(\omega+i \gamma)\right]^{\dagger} \quad(a=c),
$$

where we have added frequency arguments for clarity using the frequency relations in Eq. (26). Similarly, the damped form of Eq. (90) becomes,

$$
\begin{aligned}
& \overline{\mathbf{g}}^{a b}(-\omega+i \gamma,-\omega+i \gamma) \\
& \quad=-\left[\overline{\mathbf{g}}^{c d}(\omega+i \gamma, \omega+i \gamma)\right]^{\dagger} \quad(a=c ; \quad b=d),
\end{aligned}
$$

where we have again included frequency arguments in the right-hand side matrices for clarity. Equation (107) is the DFT equivalent of the exact damped response theory relation in Eq. (37).

We have now described how to obtain the damped TPA response function in Eq. (99) using DFT. The damped TPA function $\delta_{D}^{a b c d}(\omega)$ is then simply determined from (minus) the imaginary part of the damped TPA response function as in Eq. (48),

$$
\begin{aligned}
\delta_{D}^{a b c d}(\omega) & =-\operatorname{Im}\left[\left\langle\left\langle\overline{\mu^{a} ; \mu^{b}, \mu^{c}, \mu^{d}}\right\rangle\right\rangle_{\omega_{b}, \omega_{c}, \omega_{d}}\left(\bar{X}^{c d}\right)\right] \\
& =-\operatorname{Im}\left[\operatorname{Tr} \overline{\mathbf{g}}^{a b} \overline{\mathbf{X}}^{c d}\right],
\end{aligned}
$$

and the isotropically averaged damped TPA spectrum is calculated using Eq. (50). Due to the symmetry relations in Eqs. (106) and (107) it is necessary to solve three first-order equations (for $\bar{X}^{x}, \bar{X}^{y}$, and $\bar{X}^{z}$ ) and six second-order equations (for $\bar{X}^{x x}, \bar{X}^{y y}, \bar{X}^{z z}, \bar{X}^{x y}=\bar{X}^{y x}, \bar{X}^{x z}=\bar{X}^{z x}$, and $\bar{X}^{y z}=\bar{X}^{z y}$ ) to determine $\bar{\delta}_{D}(\omega)$. We emphasize that $\bar{\delta}_{D}(\omega)$ (nine response equations) must be determined for each optical frequency $\omega$ to calculate a full damped TPA spectrum.

\section{THE TPA SPECTRUM OF BINOL}

As a first application of damped TPA in DFT on a molecule of a relatively large size, we consider BINOL. The TPA and two-photon circular dichroism (TPCD) spectra of BINOL were reported recently ${ }^{70,71}$ for the species dissolved in tetrahydrofuran (THF). The one- and two-photon spectra of both gas-phase and solvated BINOL have also been studied computationally, see Refs. 70-72, with focus mainly on TPCD $^{74,75}$ In Ref. 72, an extensive conformational analysis was carried out, which showed that the scissoring slow vibration between the two naphthyl moieties affected negligibly the spectroscopic response of BINOL, both linear and non linear. One of the characteristics of BINOL, which makes it interesting for our first application, is that the experimental TPA spectrum, recorded between 230 and $330 \mathrm{~nm}$ (from 3.75 to $5.40 \mathrm{eV}$ ), as confirmed also by DFT calculations, ${ }^{72}$ encompasses a spectral region with a high density of excited electronic states.

The geometrical parameters used in the BINOL calculations were taken from Ref. 87 and can be found in supporting information to Ref. 72. In this work, standard and damped gas-phase TPA spectra were computed at the DFT level using the B3LYP functional. ${ }^{88-90}$ The calculations were carried out using the cc-pVDZ (670 primitives, 378 contracted) basis set ${ }^{83}$ which is expected to be of sufficient quality to

\begin{tabular}{|c|c|c|c|}
\hline \multicolumn{2}{|c|}{$\hbar \omega$} & \multirow{2}{*}{$\begin{array}{c}\lambda \\
(\mathrm{nm})\end{array}$} & \multirow{2}{*}{$\begin{array}{r}\text { TPA } \\
\text { (a.u.) }\end{array}$} \\
\hline (a.u.) & $(\mathrm{eV})$ & & \\
\hline 0.143 & 3.89 & 318 & 2.49 \\
\hline 0.144 & 3.91 & 317 & 125 \\
\hline 0.150 & 4.07 & 305 & 8.04 \\
\hline 0.150 & 4.09 & 303 & 43.8 \\
\hline 0.165 & 4.48 & 277 & 23.3 \\
\hline 0.165 & 4.49 & 276 & 55.4 \\
\hline 0.172 & 4.68 & 265 & 2.53 \\
\hline 0.172 & 4.68 & 265 & 0.829 \\
\hline 0.180 & 4.89 & 254 & 116 \\
\hline 0.178 & 4.89 & 253 & 0.418 \\
\hline 0.201 & 5.46 & 227 & 88.7 \\
\hline 0.203 & 5.52 & 225 & 222 \\
\hline 0.206 & 5.61 & 221 & 22.0 \\
\hline 0.206 & 5.62 & 221 & 1.38 \\
\hline 0.208 & 5.65 & 219 & 32.8 \\
\hline 0.208 & 5.66 & 219 & 5.30 \\
\hline 0.209 & 5.69 & 218 & 87.9 \\
\hline 0.212 & 5.77 & 215 & 85.6 \\
\hline 0.214 & 5.82 & 213 & 20.3 \\
\hline 0.216 & 5.87 & 211 & 327 \\
\hline 0.216 & 5.89 & 211 & 164 \\
\hline 0.216 & 5.89 & 211 & 3.12 \\
\hline 0.224 & 6.11 & 203 & 57.0 \\
\hline 0.224 & 6.11 & 203 & 288 \\
\hline 0.233 & 6.35 & 195 & 244 \\
\hline 0.233 & 6.35 & 195 & 323 \\
\hline 0.237 & 6.45 & 192 & 24.2 \\
\hline 0.237 & 6.46 & 192 & 54.6 \\
\hline 0.242 & 6.59 & 188 & 128 \\
\hline 0.242 & 6.59 & 188 & 104 \\
\hline 0.243 & 6.62 & 187 & 371 \\
\hline 0.244 & 6.63 & 187 & 1.83 \\
\hline 0.244 & 6.65 & 187 & 14.2 \\
\hline 0.246 & 6.69 & 185 & 23.9 \\
\hline 0.247 & 6.71 & 185 & 7.49 \\
\hline
\end{tabular}
yield a good qualitative picture of the two-photon response of
TABLE I. BINOL, cc-pVDZ basis set. Excitation energy, wavelength, and TPA absorption strengths (in a.u.). Standard TPA results. Lowest 35 excited electronic states.

BINOL, while keeping computational demands within reasonable bounds. The standard DFT eigenvalue response equations [see Eq. (95)] were solved for the lowest 35 excited electronic states and the two-photon tensors were then extracted from the single residues of the quadratic response function [see Eq. (28)]. Standard TPA results for the case of two linearly polarized photons propagating parallel to each other are given in Table I. In the damped TPA calculations, the spectrum was scanned by performing calculations using frequencies $\omega$ going from 0.03 a.u. $(0.81 \mathrm{eV}, 1520 \mathrm{~nm})$ to 0.1315 a.u. ( $3.59 \mathrm{eV}, 346 \mathrm{~nm}$ ), for a total of 28 one-photon frequencies. The damped TPA results are collected in Table II. Both the standard (sticks and convoluted) and damped TPA spectra are shown in Fig. 5. The damping factor employed for the Lorentzian used in the damped TPA calculations was $\gamma$ $=0.004$ a.u., corresponding to a HWHM of $\approx 0.10 \mathrm{eV}$. In the range of our spectra, this means HWHM going from $\approx 5$ $\mathrm{nm}($ when $\lambda=346 \mathrm{~nm})$ to $\approx 20 \mathrm{~nm}($ when $\lambda=680 \mathrm{~nm})$. All 
TABLE II. BINOL, cc-pVDZ basis set. One-photon energy, wavelength, and TPA absorption strengths (in GM). Damped TPA results with $\gamma=0.004$ a.u.

\begin{tabular}{|c|c|c|c|}
\hline \multicolumn{2}{|c|}{$\hbar \omega$} & \multirow{2}{*}{$\begin{array}{c}\lambda \\
(\mathrm{nm})\end{array}$} & \multirow{2}{*}{$\begin{array}{r}\text { TPA } \\
(\mathrm{GM})\end{array}$} \\
\hline (a.u.) & $(\mathrm{eV})$ & & \\
\hline 0.0300 & 0.8163 & 1519 & 0.000109 \\
\hline 0.0350 & 0.9524 & 1302 & 0.000192 \\
\hline 0.0400 & 1.088 & 1139 & 0.000325 \\
\hline 0.0450 & 1.225 & 1013 & 0.000542 \\
\hline 0.0500 & 1.361 & 911 & 0.000912 \\
\hline 0.0550 & 1.497 & 828 & 0.00160 \\
\hline 0.0600 & 1.633 & 759 & 0.00311 \\
\hline 0.0650 & 1.769 & 701 & 0.00806 \\
\hline 0.0700 & 1.905 & 651 & 0.0505 \\
\hline 0.0750 & 2.041 & 608 & 0.0783 \\
\hline 0.0800 & 2.177 & 570 & 0.0522 \\
\hline 0.0850 & 2.313 & 536 & 0.0665 \\
\hline 0.0900 & 2.449 & 506 & 0.161 \\
\hline 0.0950 & 2.585 & 480 & 0.0946 \\
\hline 0.1000 & 2.721 & 456 & 0.415 \\
\hline 0.1025 & 2.789 & 445 & 0.599 \\
\hline 0.1050 & 2.857 & 434 & 0.715 \\
\hline 0.1075 & 2.925 & 424 & 1.11 \\
\hline 0.1100 & 2.993 & 414 & 0.882 \\
\hline 0.1125 & 3.061 & 405 & 1.05 \\
\hline 0.1150 & 3.129 & 396 & 1.09 \\
\hline 0.1175 & 3.197 & 388 & 1.47 \\
\hline 0.1200 & 3.265 & 380 & 1.43 \\
\hline 0.1225 & 3.333 & 372 & 1.50 \\
\hline 0.1250 & 3.401 & 365 & 0.998 \\
\hline 0.1275 & 3.469 & 357 & 0.918 \\
\hline 0.1300 & 3.537 & 350 & 1.81 \\
\hline 0.1315 & 3.578 & 346 & 2.44 \\
\hline
\end{tabular}

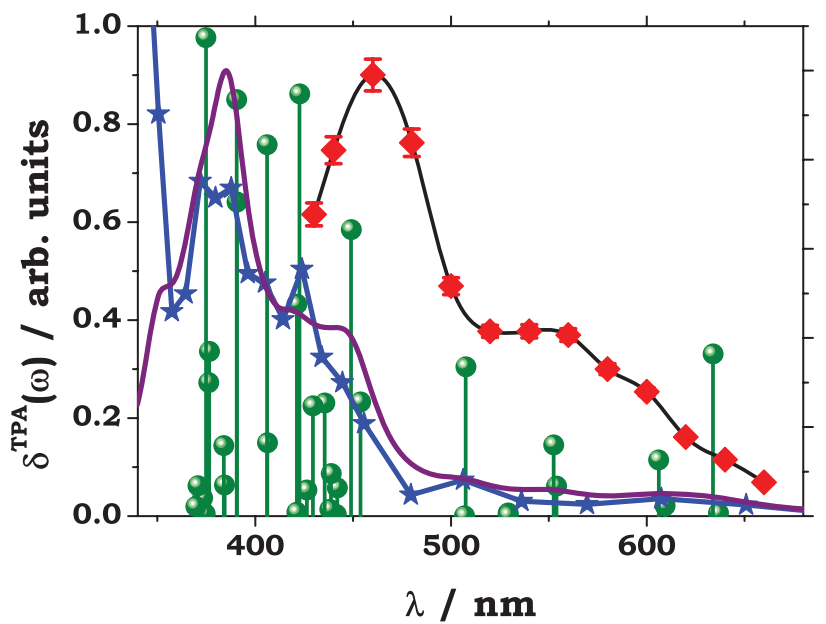

FIG. 5. BINOL, cc-pVDZ basis set. Damped $(\gamma=0.004$ a.u., blue curve with blue stars) vs. standard (vertical green sticks) TPA results. The convoluted standard spectrum, obtained with the same $\gamma$ is given by the purple curve. Also reported are the experimental data from Ref. 72, see Refs. 70 and 73. To make comparison easier, the computed damped TPA, standard convoluted and experimental spectra were all normalized in intensity. response calculations were carried out using a local version of the linear-scaling DALTON ${ }^{91}$ code.

In Fig. 5, we also report the experimental TPA data (measured in THF solution) taken from Ref. 72, obtained using the double L-scan technique developed recently in the group of Hernández, ${ }^{92}$ and discussed also in Refs. 70 and 73. The experimentally determined TPA spectrum $\delta^{\mathrm{TPA}}(\omega)$ is related to the calculated standard TPA spectrum by ${ }^{93,94}$

$$
\delta^{\mathrm{TPA}}(\omega) \approx 5.31678 \times 10^{-4} \omega^{2} \bar{\delta}_{X}(\omega),
$$

where $X=S$ for standard TPA and $X=D$ for damped TPA. Equation (109) yields the TPA spectrum in Göppert-Mayer (GM) units, when all the quantities on the right-hand side are given in atomic units. For standard TPA, $\bar{\delta}_{S}(\omega)$ is identified from Eqs. (55) and (56), with the sum in Eq. (55) restricted to the 35 first excited states (in Table I):

$$
\delta_{S}^{a b c d}(\omega)=\sum_{q=1}^{35} T_{0 q}^{a b}\left(\omega_{q} / 2, \omega_{q} / 2\right) T_{0 q}^{c d}\left(\omega_{q} / 2, \omega_{q} / 2\right) \mathcal{A}_{q}(2 \omega) .
$$

In damped TPA, $\bar{\delta}_{D}(\omega)$ is given by Eqs. (50) and (108).

The standard and damped spectra are reasonably close to each other, as they should be, except below $400 \mathrm{~nm}$, where the standard spectrum dies out abruptly, because only 35 excited states have been considered for the standard TPA spectrum, see Eq. (110). In contrast, the damped TPA spectrum by construction gets contributions from all excited states and thus also from strong TPA absorbers well below $400 \mathrm{~nm}$, which have not been determined using the standard approach. Differences between the standard and damped TPA spectra arise also elsewhere, for example around $\lambda \approx 450 \mathrm{~nm}$ where rather strong two-photon absorbing states are present, which are not seen clearly in our damped TPA spectrum due to the crude sampling of the frequency region.

Our simulated spectra show qualitatively the behavior observed in experiment, although with a net blue shift of the peak observed by experimentalists around $450 \mathrm{~nm}$ (by at least $70-75 \mathrm{~nm}$ if we assume that it can be mimicked by the relative maximum at $\approx 375 \mathrm{~nm}$ in the simulated spectra). Also, an overestimate of both the separation and the ratio in intensities between the maximum at $450 \mathrm{~nm}$ and the plateau at about $550 \mathrm{~nm}$ (experimental wavelengths) is observed. A more in depth analysis of these aspects (much along the lines of what was done in Ref. 72) goes beyond the scope of this study. In this work we have been able to show that damped response theory provides a complete and reasonable description of TPA spectra without detailed knowledge of the excited electronic state manifold and of their two-photon responses. This is of extreme importance in large molecules, where the density of excited states in regions of relevance for two-photon spectroscopy might be particularly large.

\section{CONCLUSION AND PERSPECTIVES}

We have demonstrated how TPA spectra can be calculated in damped response theory by introducing complex excitation energies into the standard response theory expressions. By construction, standard and damped TPA spectra are 
not completely identical. However, in the single-resonant case standard and damped response theory provide - for practical purposes - the same TPA spectra. In contrast, the occurrence of (approximate) double-resonances causes the standard TPA spectrum to blow up, whereas the damped TPA spectrum is well-defined and provides physically motivated TPA spectra at all optical frequencies. In particular, at exact double-resonances the damped TPA spectrum describes two successive one-photon transitions as depicted in Fig. 1(b). Even though damped TPA spectra have a more physically correct behavior at double-resonances than standard TPA spectra, it should be kept in mind that the exact appearance of the damped TPA spectra depends on an empirical broadening parameter $\gamma$, which determine the width of the absorption peaks.

The double-resonance situation becomes increasingly more common for larger molecules with dense excited state spectra, which makes damped TPA theory an attractive tool for studying the TPA of large molecules. Another argument for applying damped TPA to large molecules is their high density of states. Using standard response theory techniques ${ }^{1,85}$ each excited state must be addressed individually - regardless of its associated absorption strength. For small molecules this does not pose a problem as all absorptions in the frequency range of interest may usually be determined in this way. However, due to the very high density of the excited states in large molecules the determination of all excited states in the frequency range of interest is often a very difficult task using standard techniques. In contrast, damped TPA spectra may be obtained in any selected frequency range. A drawback of using damped TPA is of course that we only get a convoluted TPA spectrum and no information about the individual excited states. Alternatively, if the individual excitation energies and associated TPA strengths are of interest, standard and damped TPA theory can be combined: first, the largest peaks in the TPA spectrum are located using damped response theory, and subsequently individual excited states corresponding to these particular peaks are accessed in standard response theory using, for example, root homing techniques. ${ }^{95,96}$ For these reasons we consider damped response theory as a viable tool to investigate the TPA for large molecules - whether applied alone or in combination with standard response theory techniques.

\section{ACKNOWLEDGMENTS}

This work has been supported by the Lundbeck Foundation. A grant of computer time from the Norwegian Supercomputing Program is also acknowledged.

${ }^{1}$ J. Olsen and P. Jørgensen, J. Chem. Phys. 82, 3235 (1985).

${ }^{2}$ J. J. Sakurai, Modern Quantum Mechanics (Addison-Wesley, Reading, Massachusetts, 1994).

${ }^{3}$ L. Barron, Molecular Light Scattering and Optical Activity, 2nd ed. (Cambridge University Press, Cambridge, 2004).

${ }^{4}$ A. S. Davydov, Quantum Mechanics (Pergamon Press, Oxford, UK, 1965).

${ }^{5}$ B. H. Bransden and C. J. Joachain, Physics of Atoms and Molecules (Longman Group Ltd., New York, 1983).

${ }^{6}$ P. Norman, D. M. Bishop, H. J. A. Jensen, and J. Oddershede, J. Chem. Phys. 123, 194103 (2005).

${ }^{7}$ R. W. Boyd, Nonlinear Optics (Academic, San Diego, CA, 1992).
${ }^{8}$ K. Kristensen, J. Kauczor, T. Kjærgaard, and P. Jørgensen, J. Chem. Phys. 131, 044112 (2009).

${ }^{9}$ P. Norman, D. M. Bishop, H. J. A. Jensen, and J. Oddershede, J. Chem. Phys. 115, 10323 (2001).

${ }^{10}$ P. Norman, A. Jiemchooroj, and B. E. Sernelius, J. Chem. Phys. 118, 9167 (2003).

${ }^{11}$ A. Jiemchooroj, P. Norman, and B. E. Sernelius, J. Chem. Phys. 123, $124312(2005)$

${ }^{12}$ A. Jiemchooroj, P. Norman, and B. E. Sernelius, J. Chem. Phys. 125, 124306 (2006).

${ }^{13}$ X. Blase and P. Ordejón, Phys. Rev. B 69, 085111 (2004).

${ }^{14}$ L. Jensen, J. Autschbach, and G. C. Schatz, J. Chem. Phys. 122, 224115 (2005).

${ }^{15}$ R. A. Mata, B. J. C. Cabral, C. Millot, K. Coutinho, and S. Canuto, J. Chem. Phys. 130, 014505 (2009).

${ }^{16}$ T. Rocha-Rinza, K. Sneskov, O. Christiansen, U. Ryde, and J. Kongsted, Chem. Phys. Phys. Chem 13, 1585 (2011).

${ }^{17}$ P. Norman, K. Ruud, and T. Helgaker, J. Chem. Phys. 120, 5027 (2004).

${ }^{18}$ A. Jiemchooroj and P. Norman, J. Chem. Phys. 126, 134102 (2007).

${ }^{19}$ J. Autschbach, L. Jensen, and G. C. Schatz, J. Phys. Chem. A 110, 2461 (2006).

${ }^{20}$ M. Krykunov and J. Autschbach, J. Chem. Phys. 125, 034102 (2006).

${ }^{21}$ M. Krykunov, M. D. Kundrat, and J. Autschbach, J. Chem. Phys. 125, 194110 (2006).

${ }^{22}$ U. Ekström, P. Norman, V. Carravetta, and H. Ågren, Phys. Rev. Lett. 97, 143001 (2006)

${ }^{23}$ U. Ekström and P. Norman, Phys. Rev. A 74, 042722 (2006).

${ }^{24}$ G. Tu, Z. Rinkevicius, O. Vahtras, H. Ågren, U. Ekström, and P. Norman, Phys. Rev. A 76, 022506 (2007).

${ }^{25}$ A. Jiemchooroj, U. Ekström, and P. Norman, J. Chem. Phys. 127, 165104 (2007).

${ }^{26}$ A. Jiemchooroj and P. Norman, J. Chem. Phys. 128, 234304 (2008).

${ }^{27}$ P. N. M. Linares and S. Stafström, J. Chem. Phys. 130, 104305 (2009).

${ }^{28}$ M. Krykunov and J. Autschbach, J. Chem. Phys. 126, 024101 (2007).

${ }^{29}$ A. Devarajan, A. Gaenko, and J. Autschbach, J. Chem. Phys. 130, 194102 (2009).

${ }^{30}$ S. Villaume, T. Saue, and P. Norman, J. Chem. Phys. 133, 064105 (2010).

${ }^{31}$ L. Jensen, L. L. Zhao, J. Autschbach, and G. C. Schatz, J. Chem. Phys. 123, 174110 (2005).

${ }^{32}$ L. L. Zhao, L. Jensen, and G. C. Schatz, Nano Lett. 6, 1229 (2006).

${ }^{33}$ L. L. Zhao, L. Jensen, and G. C. Schatz, J. Am. Chem. Soc. 128, 2911 (2006).

${ }^{34}$ C. M. Aikens and G. C. Schatz, J. Phys. Chem. A 110, 13317 (2006).

${ }^{35}$ L. Jensen and G. C. Schatz, J. Phys. Chem. A 110, 5973 (2006).

${ }^{36}$ L. Jensen, J. Autschbach, M. Krykunov, and G. C. Schatz, J. Chem. Phys. 127, 134101 (2007).

${ }^{37}$ L. Jensen, L. L. Zhao, and G. C. Schatz, J. Phys. Chem. C 111, 4756 (2007).

${ }^{38}$ L. Jensen, C. M. Aikens, and G. C. Schatz, Chem. Soc. Rev. 37, 1061 (2008).

${ }^{39}$ Y. B. Zheng, Y.-W. Yang, L. Jensen, L. Fang, B. K. Juluri, A. H. Flood, P. S. Weiss, J. F. Stoddart, and T. J. Huang, Nano Lett. 9, 819 (2009).

${ }^{40}$ A. Mohammed, H. Ågren, and P. Norman, Chem. Phys. Lett. 468, 119 (2009).

${ }^{41}$ A. Mohammed, H. Ågren, and P. Norman, Phys. Chem. Chem. Phys. 11, 4539 (2009).

${ }^{42}$ M. Krykunov, M. Seth, T. Ziegler, and J. Autschbach, J. Chem. Phys. 127, 244102 (2007).

${ }^{43}$ H. Solheim, K. Ruud, S. Coriani, and P. Norman, J. Chem. Phys. 128, 094103 (2008).

${ }^{44}$ H. Solheim, K. Ruud, S. Coriani, and P. Norman, J. Chem. Phys. A 112, 9615 (2008)

${ }^{45}$ D. M. Bishop, J. M. Luis, and B. Kirtman, J. Chem. Phys. 116, 9729 (2002).

${ }^{46}$ P. Seidler, M. B. Hansen, W. Györffy, D. Toffoli, and O. Christiansen, J. Chem. Phys. 132, 164105 (2010).

${ }^{47}$ M. B. Hansen, P. Seidler, W. Györffy, and O. Christiansen, J. Chem. Phys. 133, 114102 (2010)

${ }^{48}$ M. Göeppert-Mayer, Ann. Phys. 9, 273 (1931).

${ }^{49}$ W. Kaiser and C. G. B. Garrett, Phys. Rev. Lett. 7, 229 (1961).

${ }^{50}$ I. D. Abella, Phys. Rev. Lett. 9, 453 (1962).

${ }^{51}$ J. H. Strickler and W. W. Webb, Proc. SPIE 1398, 107 (1991).

${ }^{52}$ W. Denk, J. H. Strickler, and W. W. Webb, Science 248, 73 (1990). 
${ }^{53}$ J. D. Bhawalkar, G. S. He, and P. N. Prasad, Rep. Prog. Phys. 59, 1041 (1996).

${ }^{54}$ P. Sperber and A. Penzkofer, Opt. Quantum Electron. 18, 381 (1986).

${ }^{55}$ D. A. Parthenopoulos and P. M. Rentzepis, Science 245, 843 (1989).

${ }^{56}$ J. H. Strickler and W. W. Webb, Opt. Lett. 16, 1780 (1991).

${ }^{57}$ F. Terenziani, C. Katan, E. Badaeva, S. Tretiak, and M. Blanchard-Desce, Adv. Mater. 20, 4641 (2008).

${ }^{58}$ G. S. He, L.-S. Tan, Q. Zheng, and P. N. Prasad, Chem. Rev. 108, 1245 (2008).

${ }^{59}$ M. Rumi and J. W. Perry, Adv. Opt. Photon. 2, 451 (2010).

${ }^{60}$ R. Moccia and A. Rizzo, J. Phys. B 18, 3319 (1985).

${ }^{61}$ E. Runge and E. K. U. Gross, Phys. Rev. Lett. 52, 997 (1984).

${ }^{62}$ M. A. L. Marques and E. K. U. Gross, Annu. Rev. Phys. Chem. 55, 427 (2004).

${ }^{63}$ E. K. U. Gross, J. F. Dobson, and M. Petersilka, "Density functional theory of time-dependent phenomena," in Topics in Current Chemistry (Springer, New York, 1996), Vol. 81.

${ }^{64}$ W. Demtröder, Laser Spectroscopy: Basic Concepts and Instrumentation 3rd ed., (Springer-Verlag, Berlin, 2002).

${ }^{65} \mathrm{~S}$. Mukamel, Principles of Nonlinear Optical Spectroscopy (Oxford University Press, Oxford, 1995).

${ }^{66}$ Y. R. Shen, The Principles of Nonlinear Optics (Wiley Interscience, New York, 1984).

${ }^{67} \mathrm{P}$. N. Butcher and D. Cotter, The Elements of Nonlinear Optics (Cambridge University Press, Cambridge, 1990).

${ }^{68}$ A. Ben-Reuven, J. Jortner, L. Klein, and S. Mukamel, Phys Rev. A 13, 1401 (1976).

${ }^{69}$ F. Gel'mukhanov, A. Baev, P. Macák, Y. Luo, and H. Ågren, J. Opt. Soc. Am. B 19, 937 (2002).

${ }^{70}$ C. Toro, L. De Boni, N. Lin, F. Santoro, A. Rizzo, and F. E. Hernández, Chem.-Eur. J. 16, 3504 (2010).

${ }^{71}$ C. Toro, L. De Boni, N. Lin, F. Santoro, A. Rizzo, and F. E. Hernández, Chirality 22, E202 (2010).

${ }^{72}$ N. Lin, F. Santoro, X. Zhao, C. Toro, L. D. Boni, F. E. Hernández, and A. Rizzo, J. Phys. Chem. B 115, 811 (2011).

${ }^{73}$ F. E. Hernández and A. Rizzo, Molecules 16, 3315 (2011).

${ }^{74}$ I. Tinoco, Jr., J. Chem. Phys. 62, 1006 (1975).

${ }^{75}$ B. Jansík, A. Rizzo, and H. Ågren, Chem. Phys. Lett. 414, 461 (2005).

${ }^{76}$ P. R. Monson and W. M. McClain, J. Chem. Phys. 53, 29 (1970).
${ }^{77}$ O. Christiansen, P. Jørgensen, and C. Hättig, Int. J. Quantum Chem. 68, 1 (1998).

${ }^{78}$ K. Sasagane, F. Aiga, and R. Itoh, J. Chem. Phys. 99, 3738 (1993).

${ }^{79} \mathrm{~K}$. Kristensen, P. Jørgensen, A. J. Thorvaldsen, and T. Helgaker, J. Chem. Phys. 129, 214103 (2008).

${ }^{80}$ Y. Yanai, D. P. Tew, and N. C. Handy, Chem. Phys. Lett. 393, 51 (2004).

${ }^{81}$ M. J. G. Peach, T. Helgaker, P. Sałek, T. W. Keal, O. B. Lutnæs, D. J. Tozer, and N. C. Handy, Chem. Phys. Phys. Chem 8, 558 (2006).

${ }^{82}$ M. J. Paterson, O. Christiansen, F. Pawłowski, P. Jørgensen, C. Hättig, T. Helgaker, and P. Sałek, J. Chem. Phys. 124, 054322 (2006).

${ }^{83}$ T. H. Dunning, Jr., J. Chem. Phys. 90, 1007 (1989).

${ }^{84}$ A. J. Thorvaldsen, K. Ruud, K. Kristensen, P. Jørgensen, and S. Coriani, J. Chem. Phys. 129, 214108 (2008).

${ }^{85}$ S. Coriani, S. Høst, B. Jansík, L. Thøgersen, J. Olsen, P. Jørgensen, S. Reine, F. Pawłowski, T. Helgaker, and P. Sałek, J. Chem. Phys. 126, 154108 (2007).

${ }^{86}$ T. Kjærgaard, P. Jørgensen, J. Olsen, S. Coriani, and T. Helgaker, J. Chem. Phys. 129, 054106 (2008).

${ }^{87}$ R. Sahnoun, S. Koseki, and Y. Fujimura, J. Mol. Struct. 735-736, 315 (2005).

${ }^{88}$ A. D. Becke, J. Chem. Phys. 98, 5648 (1993).

${ }^{89}$ A. D. Becke, Phys. Rev. A 38, 3098 (1988).

${ }^{90}$ C. Lee, W. Yang, and R. G. Parr, Phys. Rev. B 37, 785 (1988).

${ }^{91}$ DALTON Release 2.0, an ab initio electronic structure program, 2005, see http://www.kjemi.uio.no/software/dalton/dalton.html.

${ }^{92}$ L. D. Boni, C. Toro, and F. E. Hernández, Opt. Lett. 33, 2958 (2008).

${ }^{93}$ D. P. Craig and T. Thirunamachandran, Molecular Quantum Electrodynamics. An Introduction to Radiation Molecule Interaction (Dover Mineaol, New York, 1984).

${ }^{94} \mathrm{~A}$. Rizzo, S. Coriani, and K. Ruud, "Response function theory computational approaches to linear and non-linear optical spectroscopy," in Computational Strategies for Spectroscopy: from Small Molecules to Nano Systems, edited by V. Barone, (Wiley, Hoboken, NJ, 2011).

${ }^{95}$ E. R. Davidson, J. Comput. Phys. 17, 87 (1975).

${ }^{96}$ W. Butscher and W. E. Kammer, J. Comput. Phys. 20, 313 (1976).

${ }^{97}$ K. P. Huber and G. Herzberg, Molecular Spectra and Molecular Structure IV. Constants of Diatomic Molecules 1st ed. (Van Nostrand, Reinhold, New York, 1979). 